\title{
A generalized quarter car modelling approach with frame flexibility and other nonlocal effects
}

\author{
HUSAIN KANCHWALA* and ANINDYA CHATTERJEE \\ Department of Mechanical Engineering, Indian Institute of Technology, Kanpur 208016, India \\ e-mail: husain.talk@gmail.com; anindya100@gmail.com
}

MS received 14 June 2016; revised 2 November 2016; accepted 19 January 2017

\begin{abstract}
Quarter-car models are popular, simple, unidirectional in kinematics and enable quicker computation than full-car models. However, they do not account for three other wheels and their suspensions, nor for the frame's flexibility, mass distribution and damping. Here we propose a generalized quarter-car modelling approach, incorporating both the frame as well as other-wheel ground contacts. Our approach is linear, uses Laplace transforms, involves vertical motions of key points of interest and has intermediate complexity with improved realism. Our model uses baseline suspension parameters and responses to step force inputs at suspension attachment locations on the frame. Subsequently, new suspension parameters and unsprung mass compliance parameters can be incorporated, for which relevant formulas are given. The final expression for the transfer function, between ground displacement and body point response, is approximated using model order reduction. A simple Matlab code is provided that enables quick parametric studies. Finally, a parametric study and wheel hop analysis are performed for a realistic numerical example. Frequency and time domain responses obtained show clearly the effects of other wheels, which are outside the scope of usual quarter-car models. The displacements obtained from our model are compared against those of the usual quarter-car model and show ways in which predictions of the quarter-car model include errors that can be reduced in our approach. In summary, our approach has intermediate complexity between that of a full-car model and a quarter-car model, and offers corresponding intermediate detail and realism.
\end{abstract}

Keywords. Quarter-car model; laplace domain; other wheel effects; reduced order; wheel hop; frame flexibility.

\section{Introduction}

A vehicle's suspension isolates its occupants from ground disturbances. It achieves a trade-off between ride comfort and vehicle handling.

Much research has been carried out on vehicle suspensions [1]. Mathematical models for suspension design are broadly of three types: quarter-car [2], half-car [3] and fullcar models $[4,5]$. More sophisticated full-car models may include the flexibility of the frame. These models have various limitations. The quarter-car model accounts for neither the effects of three other wheels and their suspensions nor for frame flexibility. The full-car model is computationally complex. The half-car model is midway in complexity, allows for fore-aft or sideways interaction but not both, and does not incorporate frame flexibility.

Although they are popular, quarter-car models clearly cannot capture all relevant dynamic effects $[6,7]$. There are remarkable differences in ride vibration predictions of various models (quarter car, half car, half car with discrete

*For correspondence masses and models with and without frame flexibility) [8]. The engine, passenger CG locations and frame flexibility influence the vibration response, though they are not included in quarter-car model.

In the context of these range of models, our aim here is to develop a useful modelling approach of intermediate complexity, representing the car's dynamics with both reasonable accuracy and low computational effort. Our approach is depicted schematically in figure 1 .

Advantages of our proposed approach over the usual quarter-car model go beyond incorporation of frame flexibility and other-wheel effects. In a car, if we change the front left (FL) wheel suspension stiffness, we change the front right (FR) wheel suspension stiffness identically. This effect (symmetric changes at other-wheel locations) is ignored in the usual quarter-car model, but incorporated in our approach.

We note that vehicle dynamics can be modelled at different levels of complexity. For instance, in cruise control design the vehicle might be represented as a point mass [9]. For some aspects of handling, a two-wheel bicycle-like model may suffice $[10,11]$. A half-car model may elucidate 

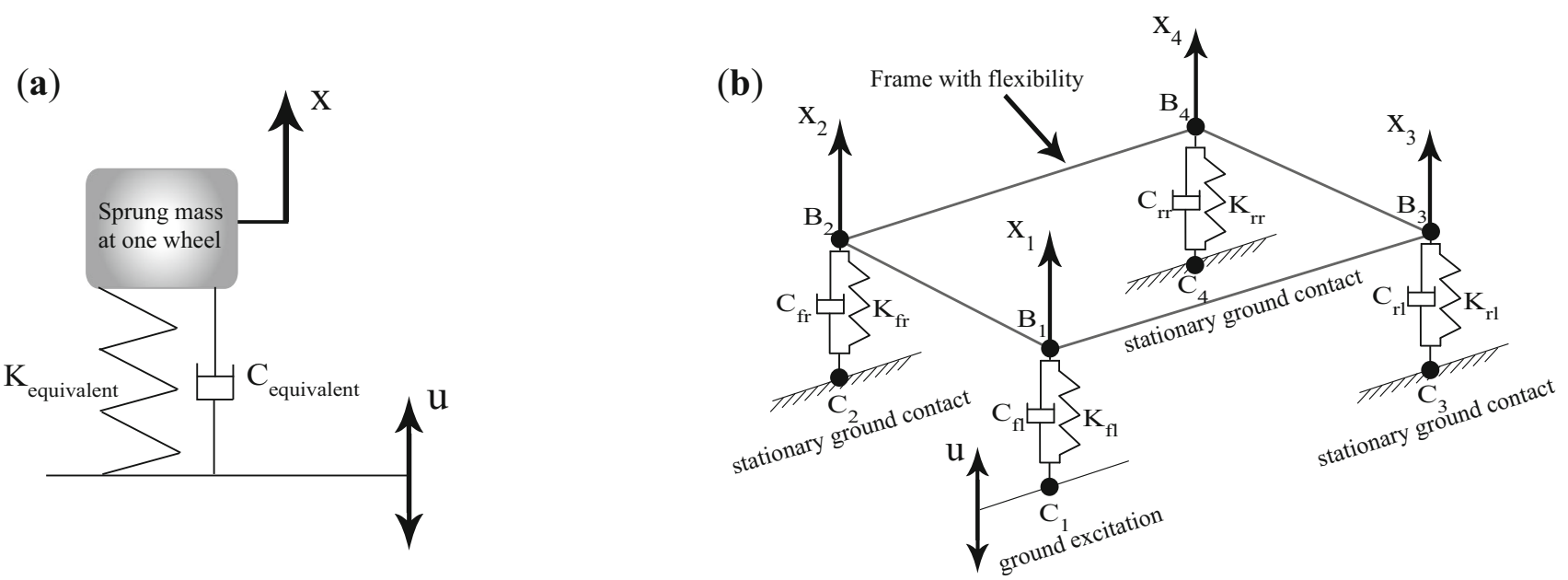

Figure 1. (a) Usual quarter-car model, with a single sprung mass. (b) Proposed approach: somewhat resembles the quarter-car model in that $x_{1}$ is computed in response to $u$, but incorporates complex dynamics of the car including effects of frame flexibility and three other wheel-ground contacts. An unsprung mass is not shown here for simplicity but will be incorporated later. We emphasize that small rolling and pitching motions of the frame are reflected in corresponding vertical motions of points $B_{i}$.

braking and stability performance $[5,12]$. In this context, our approach provides a fundamental extension of the quarter-car model, incorporating frame flexibility as well as ground-wheel contacts at three other locations.

A Matlab code for obtaining the generalized quarter-car model is provided towards the end of this paper. The code enables a user to perform fairly quick parametric studies. An example of such a parametric study is presented there as well. The role of other wheels, in particular, is seen clearly within our simple modelling framework, emphasizing the advantages of our approach over usual quarter-car models. Lastly, a numerical comparison between a usual quarter-car model and our generalized quarter-car model is given in Appendix D. As may be expected, the results reveal some inaccuracies in the usual quarter-car model, which are captured by our more general approach.

\section{Methodology}

Our proposed modelling approach is outlined using a flow chart in figure 2.

We begin with the actual vehicle in step (1) on the flow chart, which could be either a design or a prototype. A realistic vehicle model is developed using, for example, MD Adams ${ }^{\circledR}$ [13], as shown in step (2). An alternative experimental approach might, at least in principle, use direct field testing of a prototype as indicated by step (3). In such experiments, applying and then removing forces on chassis points may require less specialized equipment than a full base-excitation test, and this motivates our beginning with force inputs at chassis points $B_{i}$ as described later.

From the Adams model, it is our intention to obtain a useful transfer function matrix for the vehicle. A key point is that subsequent parameter studies should be possible without repeated Adams modelling. To this end, we identify four points on the car body $\left(B_{i} \mathrm{~s}\right)$, labelled $B_{1}-B_{4}$, where the suspension is attached (if these points are not unique, then representative points can be used). We also identify the ground contact points $\left(C_{i} \mathrm{~s}\right)$, labelled $C_{1}-C_{4}$; see subfigure (4).

Now, four independent sets of responses are calculated sequentially for unit step inputs acting one by one at the four points $B_{1}-B_{4}$. For each such step input, the displacement time histories of points $B_{1}-B_{4}$ are computed numerically. All effects of car flexibility, and the four ground contacts, are implicitly included within these computed responses. The process is indicated schematically in subfigure (4). A total of 16 different time histories are computed in this way.

These time histories are then approximated using a linear combination of decaying exponentials (plus a constant in each). The exponential rates used are the same for all 16 time histories. Sketches of four time histories are shown in subfigure (5), which shows also that the approximation can be refined when three decaying exponentials are used. These fitted exponential approximations are Laplace transformed to yield a transfer matrix $\boldsymbol{H}(\boldsymbol{s})$ between forces and displacements at points $B_{i}$ (subfigure (6)).

From $\boldsymbol{H}(\boldsymbol{s})$, we compute the transfer function matrix between ground excitation at $C_{i}$ and body displacements at $B_{i}$. A key step here is that the notional suspension parameters of the model in step (4) are now replaced by adjustable parameters in step (7). A simple optimization calculation may be conducted on the side if desired, as in step (8).

We will incorporate an unsprung mass at each wheel in the final stage (9). 


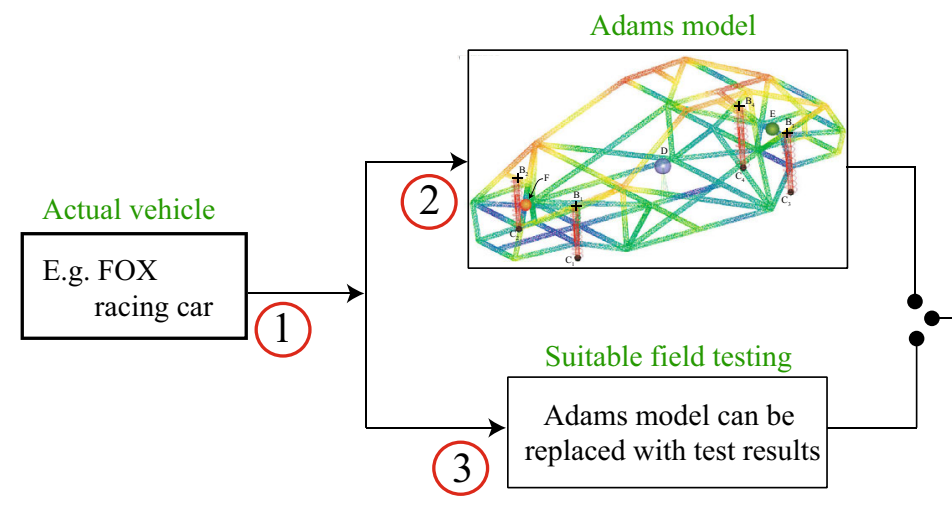

(6)

Computation of transfer matrix

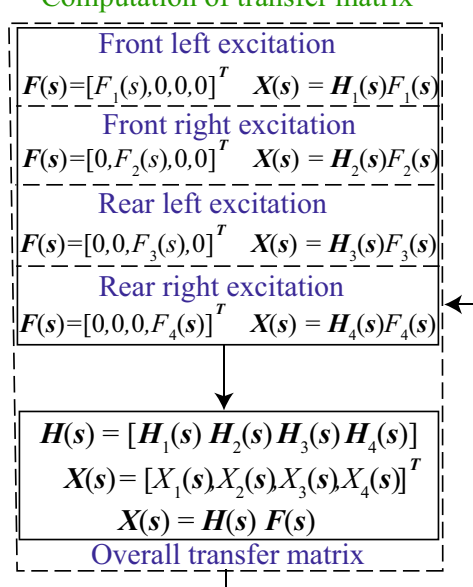

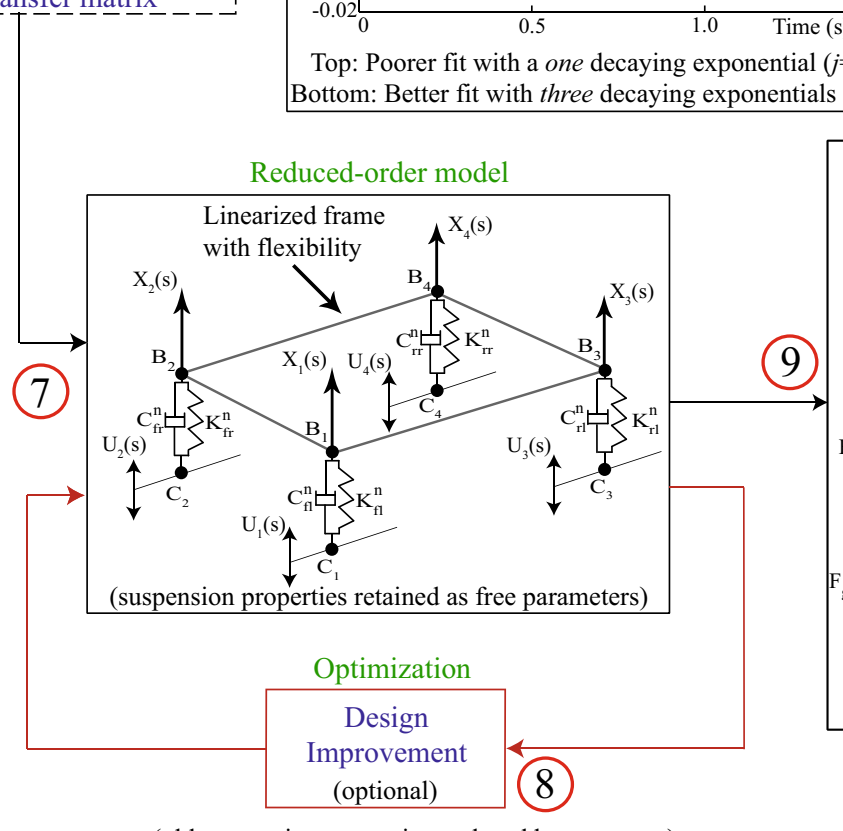

Reduced order modelling and curve fitting
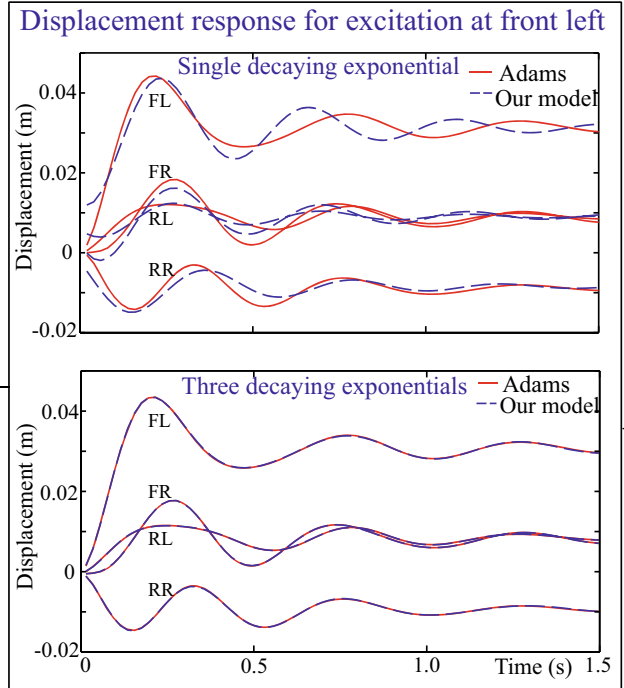

(4)

Displacement response to step excitations
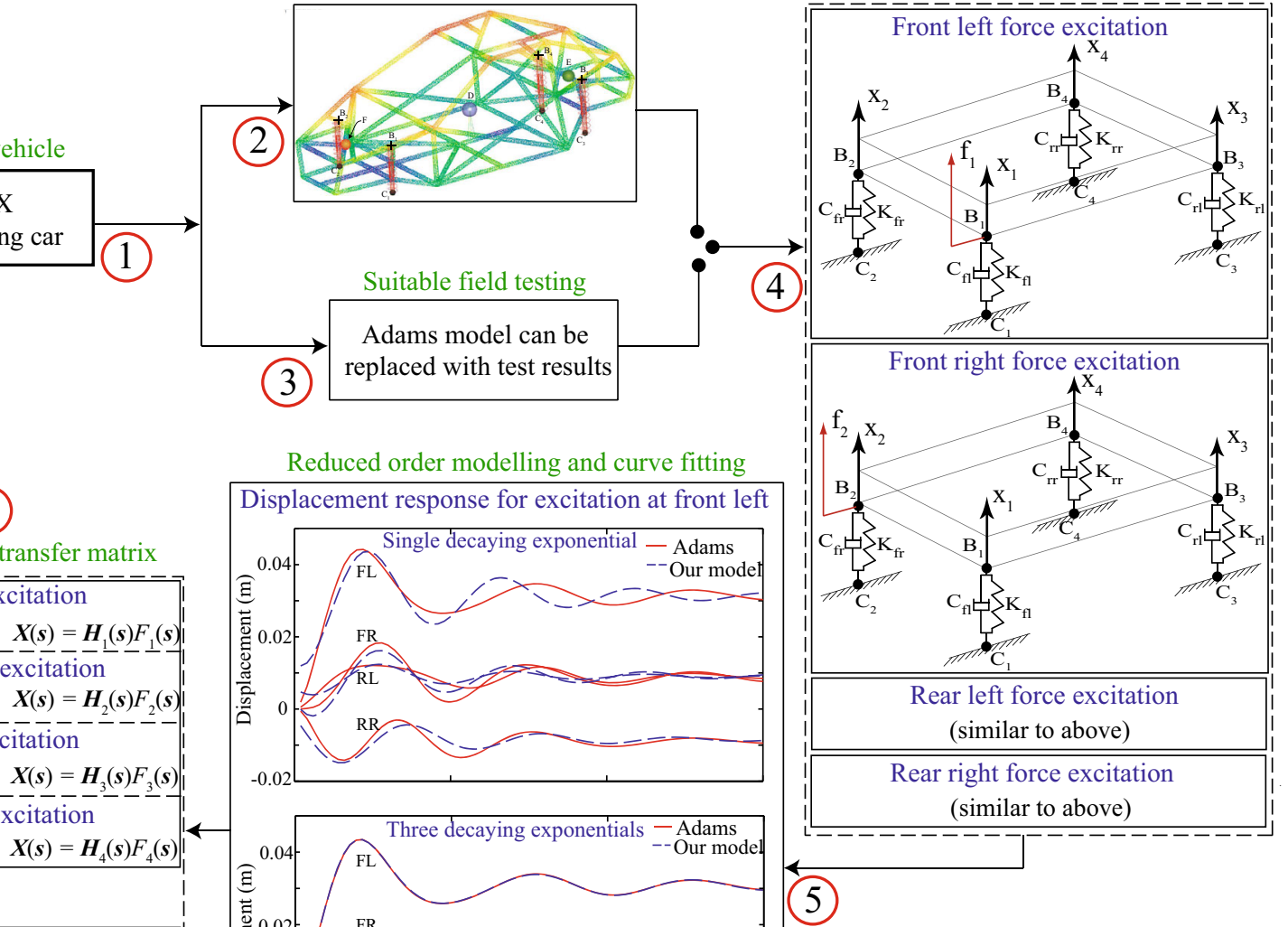

(old suspension properties replaced by new ones)

Note: Here X(s), F(s) are the Laplace transforms of $\mathrm{X}$, f respectively.

Figure 2. Work flow of this paper.

Thus, our proposed modelling approach accounts for frame flexibility and damping, details of mass distribution, as well as interactions of the wheel suspension of interest with the other three wheels' suspensions, under the simplifying assumption that the dominant ground excitation acts on the wheel of interest. In terms of kinematics, our 


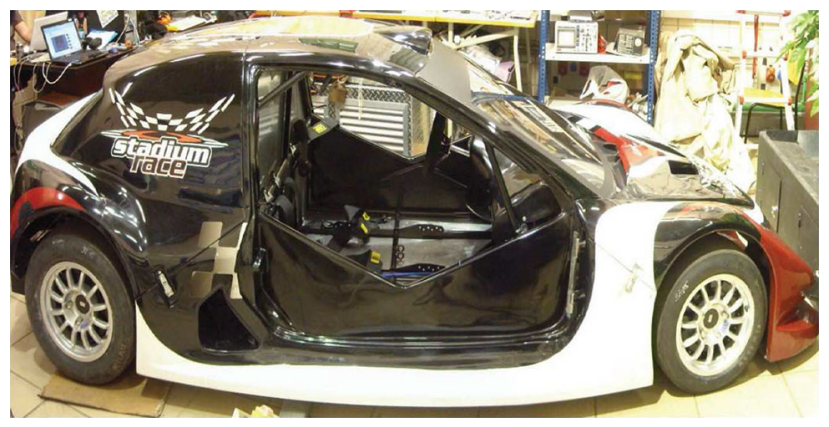

Figure 3. FOX racing car S2. Source: University of Seville, Spain.

approach retains much of the simplicity of the quarter-car model. Yet, it incorporates more realism and greater scope for parameter studies than the usual quarter-car model. At the same time, it stops short of the significantly greater complexity of full-car models, wherein parameter studies can be more laborious and time consuming.

\section{Vehicle model}

The structural part of the vehicle model used in this study is based on a FOX Silver S2 GT racing $\operatorname{car}^{1}[14,15]$. The vehicle chassis is made of a roll-cage-type structure with $1.25^{\prime \prime}$ SAE 1018 steel pipes of $0.25^{\prime \prime}$ thickness. From the CAD model of the chassis (see top-left of figure 4), a simplified but somewhat similar geometrical model was developed with pipe curvatures removed (figure 4, top right), and a finite-element (FE) model of the latter was developed in Nastran ${ }^{\circledR}$ [5]. The FE model had 17369 nodes and 18705 CQUAD4 shell elements ${ }^{2}$.

Next, a mathematical model of the vehicle including the suspension was developed in Adams ${ }^{\circledR}$. The FE model of the chassis was imported into Adams from Nastran using Adams/ Flex $^{3}$. The four mounting locations $B_{i}$ were defined as interface nodes. In addition, the model had three more interface nodes denoted D, E and F, corresponding to the centre of mass locations of the passengers, some generic payload and battery, respectively (figure 4, bottom). Three rigid bodies with mass and inertia properties representing the passengers, payload and battery were attached to these interface nodes ${ }^{4}$. In reality, flexibility and damping in the driver and seat can be

\footnotetext{
${ }^{1}$ Photograph from Carlos Bordon's laboratory in the University of Seville (figure 3), reproduced with permission.

${ }^{2}$ Four noded iso-parametric quadrilateral shell elements.

${ }^{3}$ Adams/Flex is an add-on for incorporating a component's flexibility. It uses component mode synthesis through modal superposition. We retained 42 modes for the flexible body model.

${ }^{4} \mathrm{We}$ have modelled the rigid bodies using spheres in Adams with mass of $300 \mathrm{~kg}, 100 \mathrm{~kg}, 100 \mathrm{~kg}$ and radius of gyration of $210 \mathrm{~mm}, 150 \mathrm{~mm}$, $125 \mathrm{~mm}$ respectively. The interface nodes are in turn connected to the FE mesh using RBE2 elements, which are used to connect rigid body nodes to a few nodes in a deformable mesh.
}

significant, and they have indeed been modelled elsewhere, e.g., [16]. These effects were not retained here for simplicity. It will be clear that adding such effects in our approach will simply involve adding some internal modelling details.

We now consider the vehicle suspension. The vehicle has push rod front and double wishbone rear suspensions. Effective suspension characteristics were obtained from separate Adams models of the front and rear suspension assemblies (see Appendix A). Using those simulations, the suspensions in the full-car model were replaced by four equivalent spring-dashpot pairs between the points $B_{i}$ and $C_{i}$ described earlier.

At this stage, the Adams model was complete. Forces can be applied to points $B_{i}$, and the responses of the vehicle can be computed, as depicted in subfigure (4) in figure 2 .

\section{Reduced order vehicle model}

Model order reduction has well-known advantages in largescale simulation, analysis and control design, and has been extensively studied and used. Some early papers are [17-20]. More recent work on low-order modelling in an automotive application (a quarter-car model with realistic suspension details) is reported in [21]. Here, since we are working with strongly decaying oscillatory solutions, we will directly use fitted decaying exponentials.

As discussed in section 2, we selected eight key points for reduced order modelling, namely four ground-wheel contacts $C_{i}$ and four suspension-body attachment points $B_{i}$. Consider a set of four numerical responses obtained from Adams for the motion at $B_{i}$, in response to a unit step input force $(1 \mathrm{kN})$ at $B_{1}$. The numerical responses contain discretely sampled data, at time intervals of $T=0.02 \mathrm{~s}$ in the present case. These are denoted by $x(k T)$, a $4 \times 1$ vector containing the displacements of $B_{1}-B_{4}$ at the $k^{\text {th }}$ sampling instant $^{5}$. We will approximate these displacement responses using the mathematical form

$$
\begin{aligned}
\tilde{x}(t)= & R_{0}+R_{1} e^{-\sigma_{1} t} \sin \omega_{1} t+R_{2} e^{-\sigma_{1} t} \cos \omega_{1} t+\cdots \\
& +R_{2 j-1} e^{-\sigma_{j} t} \sin \omega_{j} t+R_{2 j} e^{-\sigma_{j} t} \cos \omega_{j} t,
\end{aligned}
$$

where $\tilde{x}(t)$ is $4 \times 1$, the $R$ s are to-be-fitted $4 \times 1$ column vectors, and the $\sigma \mathrm{s}$ and $\omega \mathrm{s}$ are fitted real numbers. Equation (1) can be written more compactly as

$$
\tilde{x}(t)=R_{0}+\sum_{k=1}^{j}\left(R_{2 k-1} e^{-\sigma_{k} t} \sin \omega_{k} t+R_{2 k} e^{-\sigma_{k} t} \cos \omega_{k} t\right) .
$$

In our calculations, we initially estimated $\sigma \mathrm{s}$ and $\omega \mathrm{s}$ using a separate simple state space model, but finally refined our fits using nonlinear optimization (see Appendix B).

\footnotetext{
${ }^{5}$ The Adams solver used was GSTIFF with integrator SI2 [22].
} 

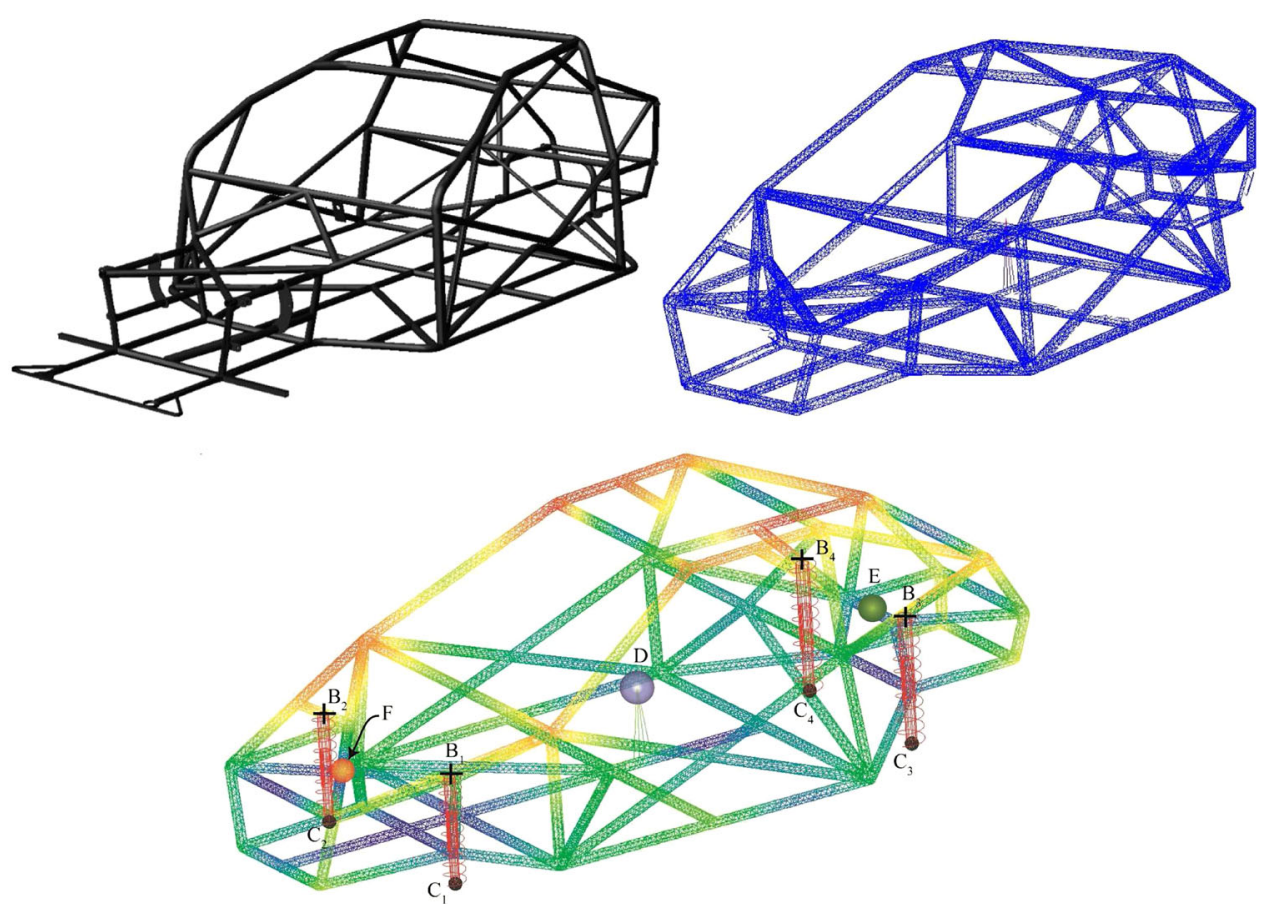

Figure 4. Top left: CAD model of the chassis. Top right: FE model of simplified chassis, Bottom: Simplified model of the car with flexible chassis and with suspension assemblies replaced by equivalent spring-dashpots.

For fitting $\sigma \mathrm{s}$ and $\omega \mathrm{s}$, we work at any time with a set of estimates for these parameters. Given these estimates, the coefficient matrices $R_{j}$ are fitted by solving the following system of equations in a least squares sense:

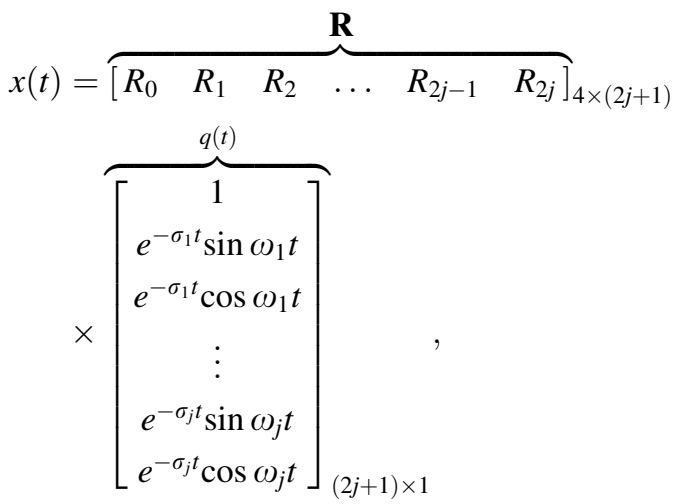

or

$$
x(t)=\mathbf{R} q(t),
$$

where $q(t)$ is a vector with a unit entry followed by exponential terms. Equation (3) is to be used for several time instants (say $N+1)^{6}$, with the left hand side vectors $x(t)$ stacked side by side to make a large $4 \times(N+1)$ matrix $\mathbf{X}$, and the right hand side vectors $q(t)$ stacked side by side to make a large $(2 j+1) \times(N+1)$ matrix $\mathbf{Q}$. Thus

\footnotetext{
${ }^{6}$ The Adams simulation was performed for $1.5 \mathrm{~s}$ with a time step $T$ of
} 0.02 s, i.e., $N=75$.

$$
\begin{aligned}
& \mathbf{X}=\left[\begin{array}{llllll}
x(0) & x(T) & \ldots & x(k T) & \ldots & x(N T)
\end{array}\right]_{4 \times(N+1)} \\
& \text { and } \\
& \mathbf{Q}=\left[\begin{array}{llllll}
q(0) & q(T) & \ldots & q(k T) & \ldots & q(N T)
\end{array}\right]_{(2 j+1) \times(N+1)}
\end{aligned}
$$

with Eq. (2) or (3) yielding

$$
\mathbf{X}=\mathbf{R Q}
$$

In Eq. (4), $\mathbf{X}$ is known from simulation (or possibly experiment, if working directly with a prototype), $\mathbf{Q}$ is known in terms of the $\sigma \mathrm{s}$ and $\omega \mathrm{s}$, and $\mathbf{R}$ is to be found. Equation (4) can be transposed and solved in a least squares sense for given $\sigma$ 's and $\omega$ 's; that sum of squares of errors is then minimized with respect to the $\sigma \mathrm{s}$ and $\omega$ s using an optimization routine (we typically use Matlab's fminsearch).

However, for our present application, this fitting needs to be simultaneously done for independent step force inputs at all suspension-to-body attachment points $B_{i}$ (16 responses in total). The same exponential rates must be used to fit all 16 responses. Thus, there are four versions of Eq. (4), one for each individual step input at four locations; and they can be stacked vertically in the form

$$
\left[\begin{array}{l}
\mathbf{X}_{1} \\
\mathbf{X}_{2} \\
\mathbf{X}_{3} \\
\mathbf{X}_{4}
\end{array}\right]_{16 \times(N+1)}=\left[\begin{array}{l}
\mathbf{R}_{1} \\
\mathbf{R}_{2} \\
\mathbf{R}_{3} \\
\mathbf{R}_{4}
\end{array}\right]_{16 \times(2 j+1)} \mathbf{Q}_{(2 j+1) \times(N+1)} .
$$


Table 1. $\sigma \mathrm{s}$ and $\omega$ s obtained for different numbers of exponentials $j$.

\begin{tabular}{lcc}
\hline No. of exponentials used $(j)$ & $\sigma$ & $\omega$ \\
\hline 1 & 3.546 & 13.894 \\
2 & 1.708 & 12.425 \\
& 4.452 & 17.042 \\
3 & 1.559 & 12.365 \\
& 3.079 & 17.257 \\
& 5.909 & 16.659 \\
\hline
\end{tabular}

In Eq. (5), $\mathbf{Q}$ does not change because the same $\sigma \mathrm{s}$ and $\omega \mathrm{s}$ are used for all four cases; the left hand side is known (computed or measured), and the matrix of $\mathbf{R s}$ is found in a least squares sense as described earlier for Eq. (4).

Results of this fitting, for different numbers of exponentials (i.e., different $j$ ), are shown in table 1 and figure 5 . The fit improves with increasing $j$, and we stop at $j=3$. Numerical values of various $R$-vectors are not reported to save space. From these fitted displacement responses, a transfer function matrix between forces and displacements at locations $B_{i}$ is developed in section 5 .

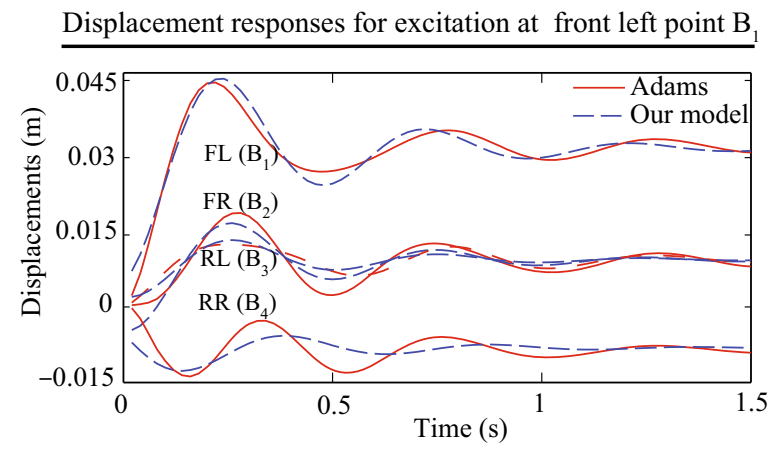

Displacement responses for excitation at rear right point $\mathrm{B}_{4}$

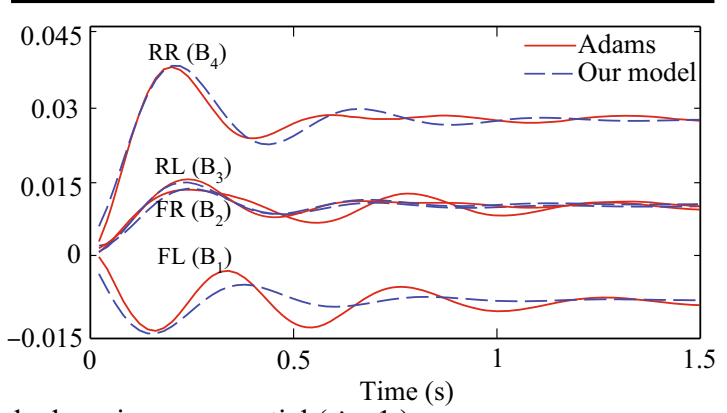

Model fitting using a single decaying exponential $(j=1)$
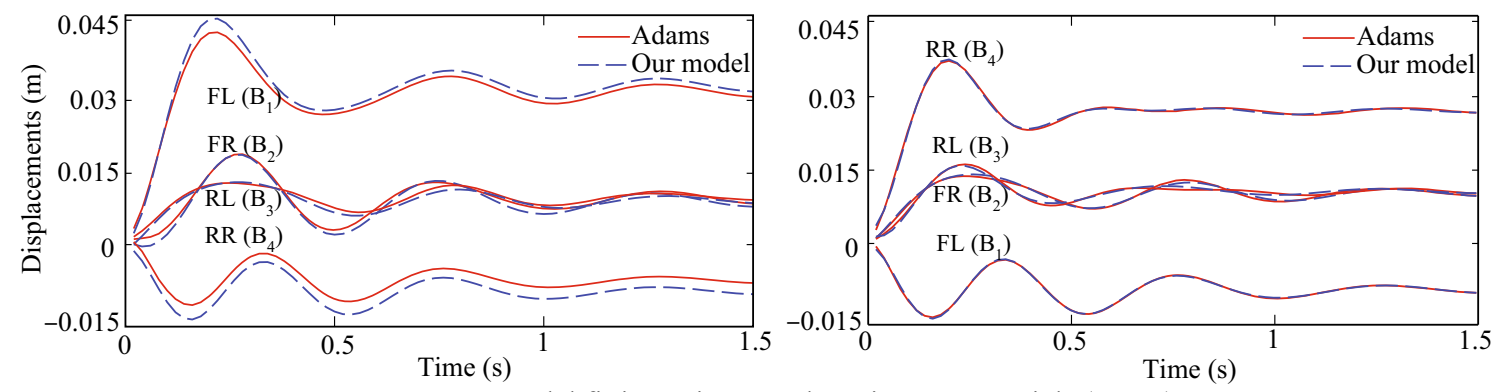

Model fitting using two decaying exponentials $(j=2)$
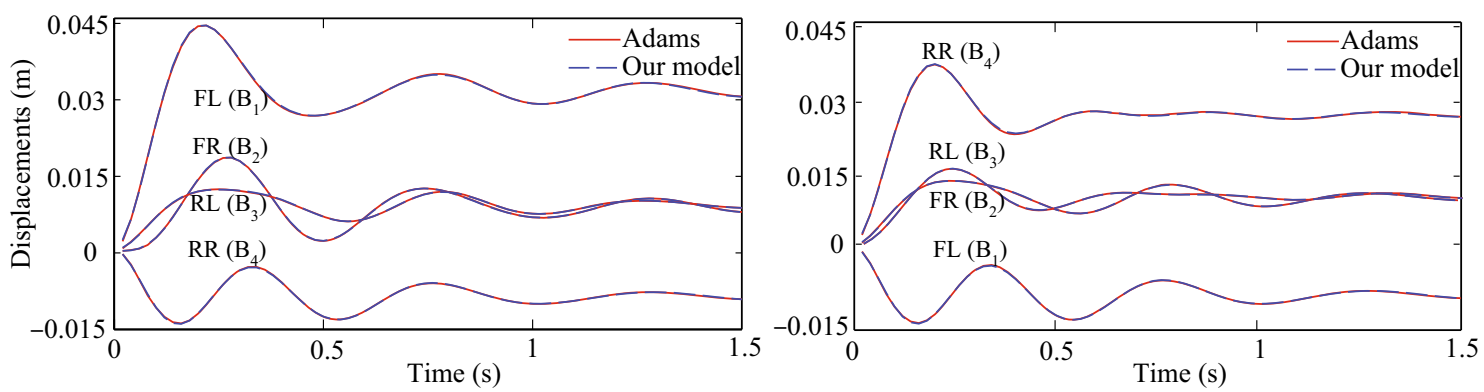

Model fitting using three decaying exponentials $(j=3)$

Figure 5. Fitted displacement responses for $j=1,2$ and 3, for two excitation cases (the other two cases are not shown). The labels are: FR for front right; FL for front left; RR for rear right; and RL for rear left. As seen clearly in the lowermost two plots, the lowest curves on both sides are identical due to the reciprocal theorem [23] applied after Laplace transformation: the response at RR due to forcing at FL equals the response at FL due to forcing at RR. The steady state displacement of the point of application of force is positive in both cases; the displacement at the diagonally opposite point is negative; and the other two displacements are smaller and about the same, because the vehicle approximately rotates about a diagonal line. Finally, displacements at the point of application are much larger than displacements at other locations, as expected. 
Note that, since $\mathbf{X}(t)$ has been explicitly fitted using exponentials, finding their Laplace transforms $(X(s))$ is simple.

\section{Transfer function matrix $H(s)$}

Assuming zero initial conditions ${ }^{7}$, the Laplace transforms of displacements, $X(s)$, and of forces, $F(s)$, at four points $B_{i}$ are related linearly as in

$$
X(s)=H(s) F(s) .
$$

Equation (6) can be expanded as

$$
\begin{aligned}
\left(\begin{array}{l}
X_{1}(s) \\
X_{2}(s) \\
X_{3}(s) \\
X_{4}(s)
\end{array}\right)= & {\left[\begin{array}{llll}
H_{11}(s) & H_{12}(s) & H_{13}(s) & H_{14}(s) \\
H_{12}(s) & H_{22}(s) & H_{23}(s) & H_{24}(s) \\
H_{13}(s) & H_{23}(s) & H_{33}(s) & H_{34}(s) \\
H_{14}(s) & H_{24}(s) & H_{34}(s) & H_{44}(s)
\end{array}\right] } \\
& \times\left(\begin{array}{l}
F_{1}(s) \\
F_{2}(s) \\
F_{3}(s) \\
F_{4}(s)
\end{array}\right) . \\
\uparrow & \uparrow
\end{aligned}
$$

Each element of the above $4 \times 4$ matrix is a separate Laplace transform, and individual columns have been named $H_{1}(s)-H_{4}(s)$ as shown.

First, a step input force is applied at point $B_{1}$, with all other input forces equal to zero. The Laplace transform of the force input is then $F_{1}(s)=\frac{1}{s}$, with $F_{2}=F_{3}=F_{4}=0$. The corresponding response $X(s)$ is computed analytically from the fitted exponentials as described earlier; this same $X(s)$ is then (by Eq. (6)) equal to $H_{1}(s) F_{1}(s)$, whence $H_{1}(s)=X(s) F_{1}(s)^{-1}=s X(s)$. In this way, with four successive simulation results, all the columns of $H(s)$ are found.

We expect from theory that $H(s)$ can be taken as symmetric, as follows. The linearized dynamics of the vehicle, subjected to vertical forces at four locations $\left(B_{i} \mathrm{~s}\right)$, can generally be described by a model of the form

$$
\begin{gathered}
{\left[\begin{array}{cc}
M_{11} & M_{12} \\
M_{12}{ }^{T} & M_{22}
\end{array}\right]\left[\begin{array}{l}
\ddot{x} \\
\ddot{y}
\end{array}\right]+\left[\begin{array}{cc}
C_{11} & C_{12} \\
C_{12}{ }^{T} & C_{22}
\end{array}\right]\left[\begin{array}{c}
\dot{x} \\
\dot{y}
\end{array}\right]+\left[\begin{array}{cc}
K_{11} & K_{12} \\
K_{12}{ }^{T} & K_{22}
\end{array}\right]} \\
{\left[\begin{array}{l}
x \\
y
\end{array}\right]=\left[\begin{array}{l}
f \\
0
\end{array}\right] .}
\end{gathered}
$$

Here, $x$ represents the vertical displacements at $B_{1}-B_{4}$, and $y$ represents a possibly very large number of additional

\footnotetext{
${ }^{7}$ We have assumed zero initial conditions as we are interested in the response of the vehicle under sustained road inputs (disturbances) in which the role of initial conditions rapidly becomes insignificant.
}

unmeasured and unforced degrees of freedom ${ }^{8} . M$ and $K$ are respectively, symmetric mass and stiffness matrices; their symmetry is a consequence of the quadratic form of the kinetic and potential energies in Lagrange's formulation. The damping matrix $C$ is also symmetric in typical formulations. The reason for the symmetry of $C$ is that our dynamic model of the vehicle frame consists of (i) assigned modal damping values for the structure (automatically giving symmetric damping matrices), along with (ii) added discrete dashpots in the suspension, which make symmetric contributions given by Rayleigh's dissipation function [24]. Additionally, and more generally, several other linear damping models can in fact be accurately captured using symmetric matrices, as discussed in, e.g., [25, 26].

Taking the Laplace transform of Eq. (8) we obtain

$$
\begin{gathered}
{\left[\begin{array}{cc}
M_{11} & M_{12} \\
M_{12}{ }^{T} & M_{22}
\end{array}\right]\left[\begin{array}{c}
X(s) \\
Y(s)
\end{array}\right] s^{2}+\left[\begin{array}{cc}
C_{11} & C_{12} \\
C_{12}{ }^{T} & C_{22}
\end{array}\right]\left[\begin{array}{c}
X(s) \\
Y(s)
\end{array}\right] s} \\
+\left[\begin{array}{cc}
K_{11} & K_{12} \\
K_{12}{ }^{T} & K_{22}
\end{array}\right]\left[\begin{array}{c}
X(s) \\
Y(s)
\end{array}\right]=\left[\begin{array}{c}
F(s) \\
0
\end{array}\right],
\end{gathered}
$$

rewritten compactly as

$$
\left[\begin{array}{cc}
G_{11}(s) & G_{12}(s) \\
G_{12}(s)^{T} & G_{22}(s)
\end{array}\right]\left[\begin{array}{c}
X(s) \\
Y(s)
\end{array}\right]=\left[\begin{array}{c}
F(s) \\
0
\end{array}\right]
$$

whence, eliminating $Y(s)$, we obtain $X(s)=H(s) F(s)$ with

$$
H(s)=\left[G_{11}(s)-G_{12}(s) G_{22}(s)^{-1} G_{12}(s)^{T}\right]^{-1},
$$

which is symmetric. Since $H(s)$ is symmetric, the system obeys reciprocity (see also, e.g., [23]). As a result, the response at RR (rear-right) due to unit forcing at FL (front-left) equals the response at FL due to unit forcing at RR, as seen in figure 5.

In the next section we obtain the transfer function matrix $H_{n}(s)$ between ground excitations at $C_{i}$ and displacements at $B_{i}$.

\section{Modified transfer function matrix $H_{n}(s)$}

The matrix $H(s)$ relates forces and displacements at points $B_{i}$. In determining $H(s)$, a set of baseline suspension properties were used. If we change the suspension properties during subsequent simulations, we do not wish to repeat the process of exponential fitting. Instead, we will modify $H(s)$ to incorporate new suspension properties treated as free parameters. Subsequently, we will use the modified $H(s)$ to determine a matrix $H_{n}(s)$ that relates displacement inputs at points $C_{i}$ to displacements at points $B_{i}$.

\footnotetext{
${ }^{8}$ The FE model of the chassis has 17369 nodes. Four of them correspond to points $B_{1}$ through $B_{4}$. The non-vertical displacements of these four points, and the displacements of all remaining nodes, are unforced and unmeasured degrees of freedom.
} 


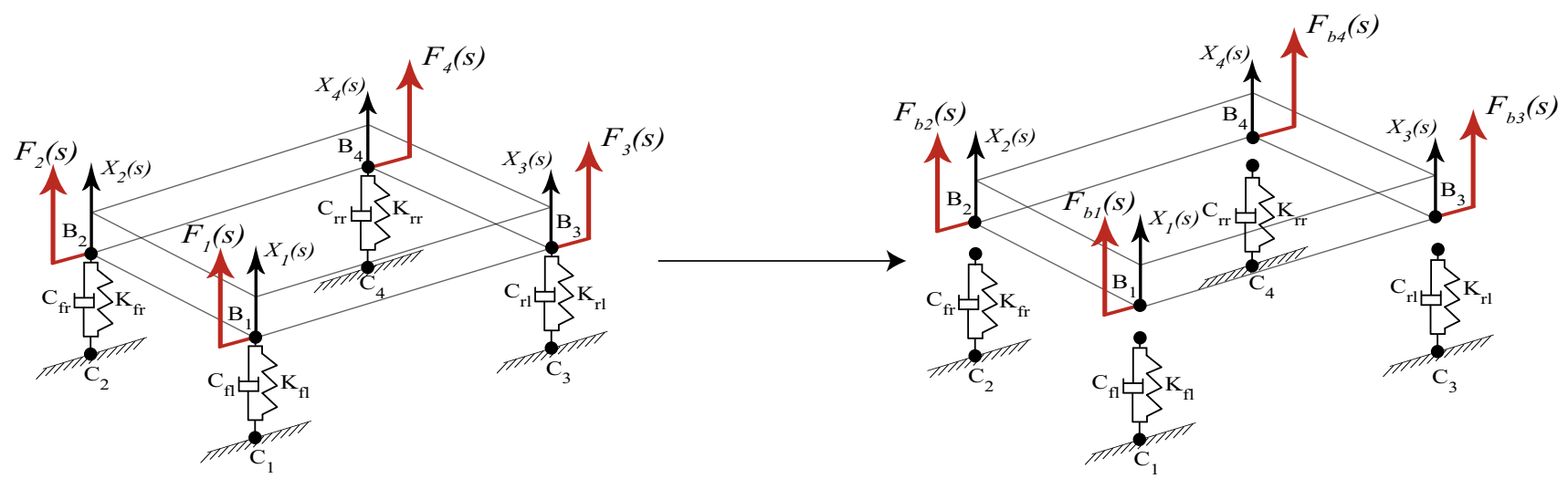

Figure 6. Towards finding $H_{f}(s)$ with suspension properties retained as free parameters.

\subsection{Incorporating suspension properties as free parameters}

We have initially applied forces $F(s)$ at $B_{i}$ and obtained $X(s)$ using $H(s)$. The relationship can be formally inverted (displacements to required additional forces) as in

$$
X(s)=H(s) F(s) \longrightarrow F(s)=H(s)^{-1} X(s) .
$$

To obtain $H_{f}(s)$, we first imagine detaching the suspensions (spring-dashpots) from the vehicle body, yet applying the same displacements $X(s)$ at points $B_{i}$ (see figure 6).

With suspensions detached, the required additional forces at $B_{i}$ will change from $F(s)$ to $F_{b}(s)$ as in

$$
F_{b}(s)=F(s)-D_{o}(s) X(s),
$$

where the subscript ' $o$ ' denotes 'old' or baseline properties, subscript ' $b$ ' denotes 'body' and the diagonal matrix

$$
D_{o}(s)=\left[\begin{array}{cccc}
K_{f l}+C_{f l} s & 0 & 0 & 0 \\
0 & K_{f r}+C_{f r} s & 0 & 0 \\
0 & 0 & K_{r l}+C_{r l} s & 0 \\
0 & 0 & 0 & K_{r r}+C_{r r} s
\end{array}\right]
$$

where in turn $K_{f l}, K_{f r}, K_{r l}, K_{r r}$ are the equivalent stiffnesses and $C_{f l}, C_{f r}, C_{r l}, C_{r r}$ are the corresponding damping coefficients of FL, FR, RL and RR wheel-suspension assemblies respectively. We assume for simplicity that the left and right suspension properties are identical (lateral symmetry).

Now, if we replace $D_{o}(s)$ with $D_{n}(s)$ (' $n$ ' denotes 'new' suspension properties $)^{9}$, the forces in response to the same $X(s)$ will become (using Eqs. (13) and (12))

\footnotetext{
${ }^{9}$ The suspension properties used for illustration are given in table 2 . The original suspension parameters were obtained from the Adams model of the vehicle (see Appendix A). The new suspension parameter values were obtained from a peripheral optimization study (see (8) in figure 2) that is not relevant here.
}

Table 2. Original and new suspension parameters.

\begin{tabular}{lcccc}
\hline Identifier & $K_{f}(\mathrm{~N} / \mathrm{mm})$ & $K_{r}(\mathrm{~N} / \mathrm{mm})$ & $C_{f}(\mathrm{Ns} / \mathrm{mm})$ & $C_{r}(\mathrm{Ns} / \mathrm{mm})$ \\
\hline Original & 25.0 & 30.0 & 0.50 & 0.75 \\
New & 23.6 & 34.9 & 0.62 & 0.76 \\
\hline
\end{tabular}

$$
\begin{aligned}
& F_{n}(s)=F_{b}(s)+D_{n}(s) X(s)=\left[H(s)^{-1}-D_{o}(s)+D_{n}(s)\right] \\
& X(s) .
\end{aligned}
$$

Thus, upon changing suspension parameters, we obtain a new transfer function $H_{f}(s)$ to replace the old $H(s)$ as

$$
X(s)=\underbrace{\left[H(s)^{-1}-D_{o}(s)+D_{n}(s)\right]^{-1}}_{H_{f}(s)} F_{n}(s) .
$$

The matrix $H_{f}(s)$ obtained above (' $f$ ' denoting free parameters), between forces and displacements at $B_{i}$, corrects the original $H(s)$ for changed suspension parameters. The transfer function matrix $H_{f}(s)$ may be useful in future studies where nonlinearity is to be introduced in one wheel's suspension. Here, however, we continue our linear formulation and consider ground displacement inputs.

\subsection{Obtaining the transfer function matrix from ground to body displacements}

We now incorporate displacement inputs at ground-contact points $C_{i}$ (figure 7). To this end, recall the case without ground inputs, i.e., Eq. (15). If displacements $U(s)$ are additionally applied to the ground contact points, while holding $X(s)$ constant, then additional forces $D_{n}(s) U(s)$ are transmitted to the contact points $B_{i}$. For $X(s)$ to remain the same in Eq. (15), we must subtract $D_{n}(s) U(s)$ from $F_{n}(s)$. This leads to 


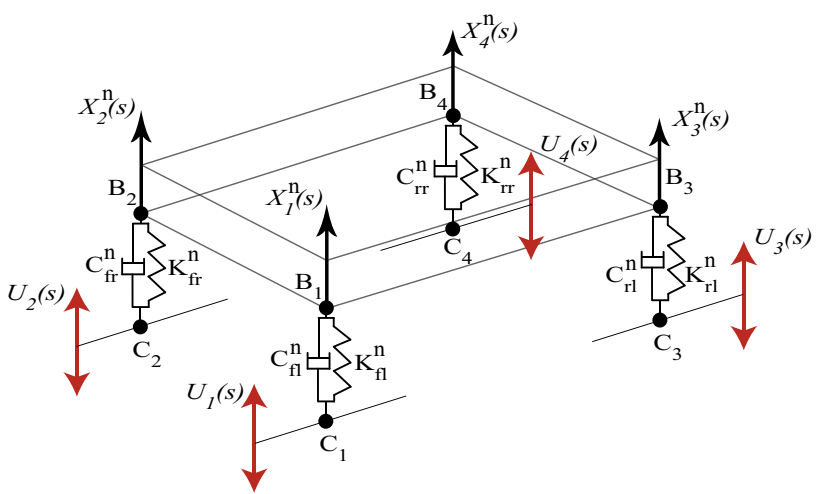

Figure 7. Displacement inputs at ground contact points $C_{i}$.

$$
F_{w g i}(s)=\left[H(s)^{-1}-D_{o}(s)+D_{n}(s)\right] X(s)-D_{n}(s) U(s),
$$

where the 'wgi' subscript stands for 'with ground input.' When there is only base excitation from the ground, and no additional forces are applied, the left hand side above becomes zero and we have

$$
X(s)=\underbrace{\left[H(s)^{-1}-D_{o}(s)+D_{n}(s)\right]^{-1} D_{n}(s)}_{H_{n}(s)} U(s),
$$

where $H_{n}(s)$ is the transfer function matrix between displacements at $C_{i}$ and $B_{i}$ (' $n$ ' denotes 'new' suspension properties).

\section{Model with unsprung mass}

The term 'unsprung mass' is attributed to Healey [27]. It refers to the inertial effects of the suspension, wheels and other components directly connected to them, rather than the mass supported by the suspension. We will now incorporate an unsprung mass in our formulation by attributing an effective mass to the wheel ${ }^{10}$.

A schematic diagram of one wheel including its unsprung mass is shown in figure 8. The key point is that, due to the nonzero mass, the force from the ground is not transmitted directly through the wheel to the base of the suspension. In terms of figure $8, F_{g, i} \neq F_{t, i}$.

The $i^{\text {th }}$ wheel's suspension now relates four dynamic variables: displacement $U_{g, i}(s)$ and corresponding force $F_{g, i}(s)$ at the true ground contact, and the transmitted displacement and force $U_{t, i}(s)$ and

\footnotetext{
${ }^{10}$ Sometimes point masses are also added to the body points $B_{i}$, but that does not affect our procedure.
}

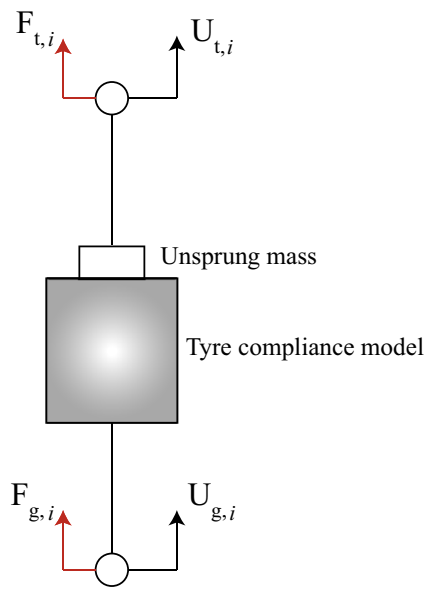

Figure 8. Schematic diagram representing dynamic compliance of $i^{\text {th }}$ wheel including its 'unsprung' mass. Here, $U_{t, i}(s)$ corresponds to the base displacement $U(s)$ in Eq. (18).

$F_{t, i}(s)$, respectively, at the suspension base point (they replace the points called $C_{i}$ earlier). In other words, $U_{t, i}(s)$ is the same as the $i^{\text {th }}$ component of displacement $U(s)$ in Eq. (18).

These forces and displacements are related by

$$
\left[\begin{array}{l}
F_{g, i}(s) \\
F_{t, i}(s)
\end{array}\right]=\underbrace{\left[\begin{array}{ll}
T_{11}(s) & T_{12}(s) \\
T_{21}(s) & T_{22}(s)
\end{array}\right]} \quad\left[\begin{array}{l}
U_{g, i}(s) \\
U_{t, i}(s)
\end{array}\right],
$$

Tyre's dynamic compliance

where $i=1,2,3$ and 4 for FL, FR, RL, and RR wheel, respectively. It is clear that these individual suspensions' dynamic compliances are unaffected by phenomena at other wheels. Considering the FL wheel we have

$$
F_{t, 1}(s)=D_{n, 11}(s)\left(X_{1}(s)-U_{t, 1}(s)\right) .
$$

From Eqs. (19) and (20), we obtain

$$
D_{n, 11}(s)\left(X_{1}(s)-U_{t, 1}(s)\right)=T_{21}(s) U_{g, 1}(s)+T_{22}(s) U_{t, 1}(s)
$$

which yields

$$
\begin{aligned}
U_{t, 1}(s)= & \left(D_{n, 11}(s)+T_{22}(s)\right)^{-1} D_{n, 11} X_{1}(s) \\
& -\left(D_{n, 11}(s)+T_{22}(s)\right)^{-1} T_{21}(s) U_{g, 1}(s) .
\end{aligned}
$$

Equation (21) is of the form

$$
U_{t, 1}(s)=\bar{A}_{1}(s) X_{1}(s)-\bar{B}_{1}(s) U_{g, 1}(s) .
$$

Four versions of this equation, one for each wheel, can be assembled in matrix form as 


$$
\begin{aligned}
U_{t} & =\underbrace{\left[\begin{array}{cccc}
\bar{A}_{1}(s) & 0 & 0 & 0 \\
0 & \bar{A}_{2}(s) & 0 & 0 \\
0 & 0 & \bar{A}_{3}(s) & 0 \\
0 & 0 & 0 & \bar{A}_{4}(s)
\end{array}\right]}_{A(s)} \\
X & -\underbrace{\left[\begin{array}{cccc}
\bar{B}_{1}(s) & 0 & 0 & 0 \\
0 & \bar{B}_{2}(s) & 0 & 0 \\
0 & 0 & \bar{B}_{3}(s) & 0 \\
0 & 0 & 0 & \bar{B}_{4}(s)
\end{array}\right]}_{B(s)} U_{g}(s) .
\end{aligned}
$$

From Eq. (18), noting that $U_{t}(s) \equiv U(s)$, we have

$$
U_{t}(s)=H_{n}^{-1}(s) X(s)
$$

which finally yields

$$
X(s)=\left[A(s)-H_{n}^{-1}(s)\right]^{-1} B(s) U_{g}(s)=G(s) U_{g}(s),
$$

where $U_{g}(s)$ is the actual base excitation from the true ground-contact point. As a check we may note that if the tyres' compliance is set to zero, then in Eq. (23) we must have $A(s)=0$ and $B(s)=I$ (the identity matrix), whence Eq. (25) reduces to Eq. (18).

The resulting car model is shown schematically in figure 9. Superficially, it looks like a full-car model, but it is full-car restricted in various ways. For example, the motion kinematics at the body points $B_{i}$ is purely vertical. Moreover, the development of the model is based on individual attention to one-dimensional behaviours of individual wheels' suspensions. A desirable practical use of this model lies in setting input ground displacements at three wheels to zero, and taking the appropriate scalar diagonal element of

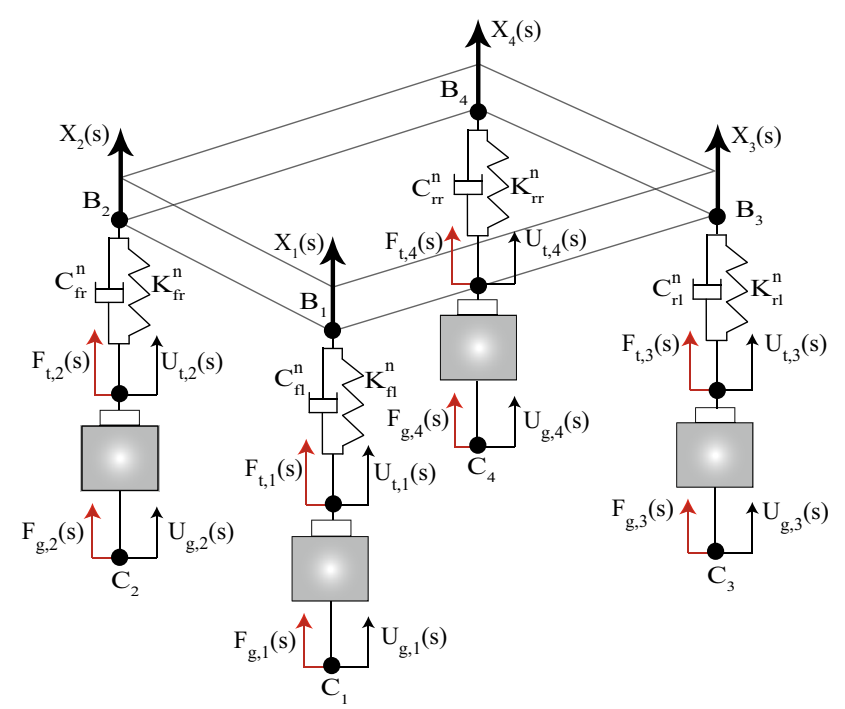

Figure 9. Car model with unsprung mass.
Table 3. Parameter values used in the unsprung mass compliance model. Note that the unsprung mass is small compared with the total sprung mass (548 $\mathrm{kg}$ in our case); the stiffness is large and the damping is small compared with the suspension parameters of table 2 . The unsprung mass values used are high for conventional wheels. These values are used for clearer demonstration later, and are representative of wheels with hub motors.

\begin{tabular}{lccc}
\hline Identifier & $M_{u}$ (unsprung) & $K_{t}$ (tyre) & $C_{t}$ (tyre) \\
\hline Front & $45 \mathrm{~kg}$ & $250 \mathrm{~N} / \mathrm{mm}$ & $0.05 \mathrm{Ns} / \mathrm{mm}$ \\
Rear & $50 \mathrm{~kg}$ & $275 \mathrm{~N} / \mathrm{mm}$ & $0.06 \mathrm{Ns} / \mathrm{mm}$ \\
\hline
\end{tabular}

$G(s)$ in Eq. (25), to obtain the resulting quarter-car model for the wheel of interest, while incorporating chassis flexibility and other-wheel effects to a useful extent.

For practical work, the matrix manipulations leading to Eq. (25) lead to long analytical expressions that can be simplified without sacrificing accuracy as shown below.

\section{Model order reduction of the final transfer function matrix $G(s)$}

Model order reduction of $G(s)$ leads to obvious computational simplifications.

We have already done order reduction through direct exponential fitting in section 4 to obtain $H(s)$ using three pairs of decaying sines and cosines $(j=3$ in table 1$)$ plus an added constant. Consequently, each element of $H(s)$ is a transfer function with both numerator and denominator of $6^{\text {th }}$ order. The subsequent matrix operations with $H(s)$, as described in Eqs. (12)-(18), can be done symbolically in Matlab. The matrix $H_{n}(s)$ of Eq. (18) has elements of $28^{\text {th }}$ order, which is quite large.

Finally, $H_{n}(s)$ is itself modified using Eqs. (19)-(25) to incorporate an unsprung mass compliance model (see table 3), leading to a more realistic vehicle transfer function matrix $G(s)$ as in Eq. (25).

The elements of $G(s)$ are of $36^{\text {th }}$ order. One of them is reproduced for illustration in Appendix C. Such complicated expressions can be simplified for easier use. We are primarily interested in one diagonal element of $G(s)$, e.g., $G_{11}(s)$ for the FL wheel; accordingly, we demonstrate the reduction of $G_{11}(s)$.

Since $G_{11}(s)$ is a $36^{\text {th }}$ order transfer function, its equivalent time domain model will have 36 states, given by, $\left[x_{1}(t), \dot{x}_{1}(t), \ddot{x}_{1}(t), \ldots, x_{1}{ }^{(36)}(t)\right]$. Different reduced order models were developed using Matlab's built-in function balred ${ }^{11}$. A comparison of these reduced order models is shown in figure 10 .

Figure 10 shows that a $6^{\text {th }}$ order approximation $G_{\text {red }}(s)$ preserves the model characteristics accurately. The frequency response and the time domain step-input response

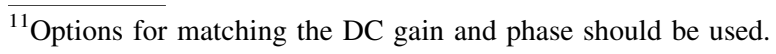


Bode diagram
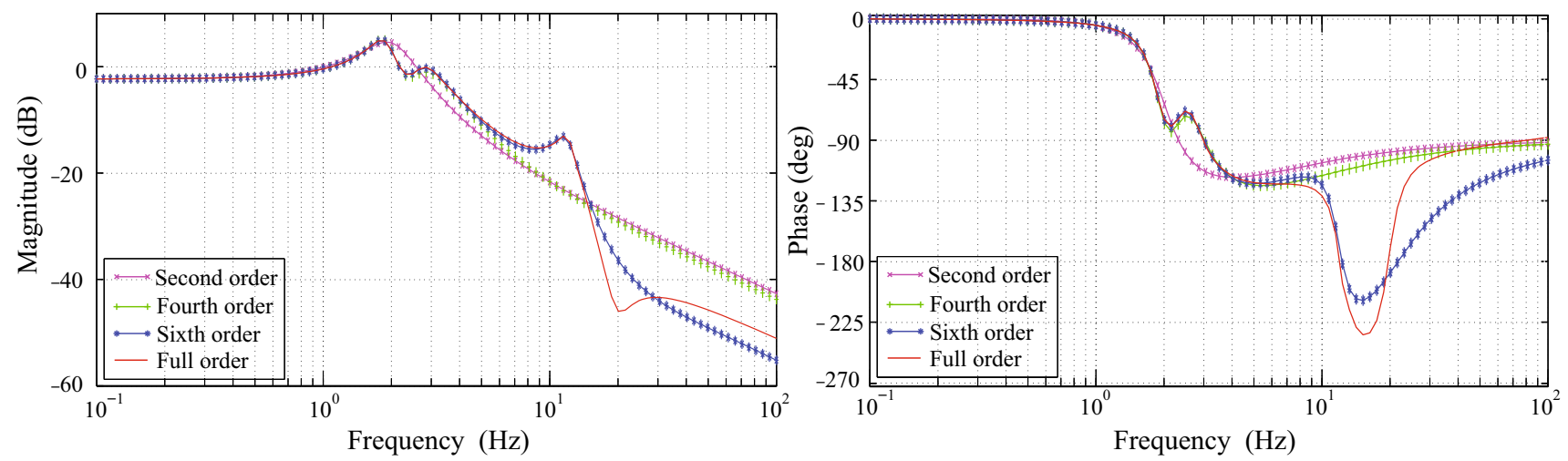

Figure 10. Bode plot comparison of various reduced order models against the full order model.
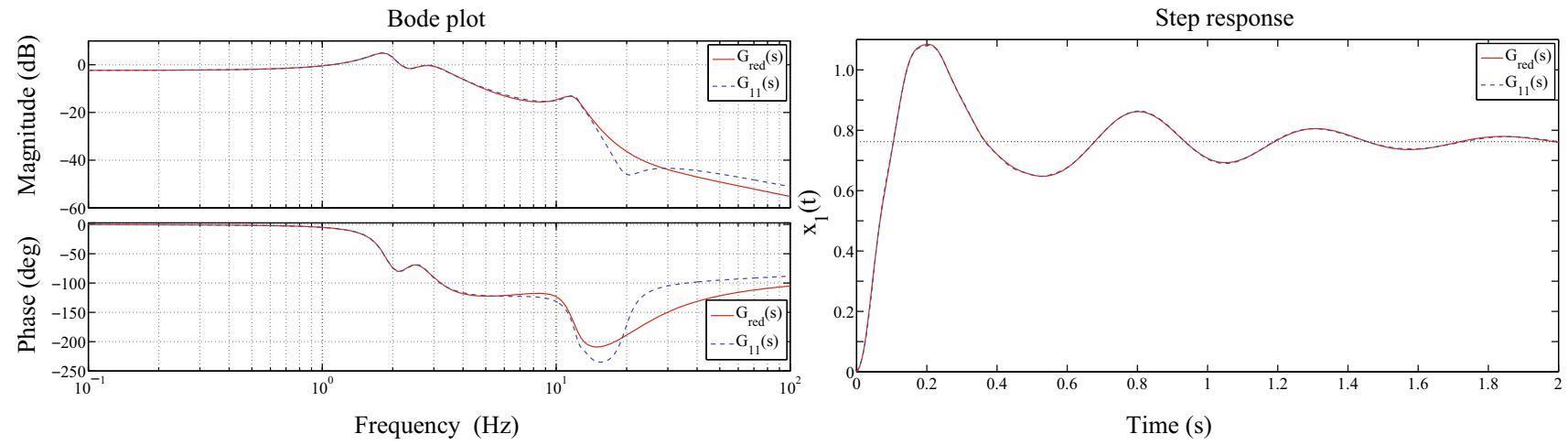

Figure 11. Comparison of frequency domain response (Bode plot: left) and time domain response (step response: right) of the reduced order $G_{r e d}(s)$ (solid) and the original $G_{11}(s)$ (dashed) transfer function model.

of the reduced order $G_{r e d}(s)$ and the original $G_{11}(s)$ are compared in figure 11 . The match is good in the frequency range plotted (up to $100 \mathrm{~Hz}$ ). In particular, on a logarithmic scale, differences are seen only where the response itself is negligibly small.

There are three peaks in the Bode plot of figure 11. The first and second peaks correspond to the front and rear suspension natural frequencies $(1.8$ and $2.6 \mathrm{~Hz})$ and the third peak represents the wheel hop frequency $(10.3 \mathrm{~Hz})$.

\section{Recipe for Matlab implementation of the proposed approach}

Finally, we provide the Matlab code for simple implementation of the entire procedure for obtaining the generalized quarter-car model, beginning from the original Laplace transforms of Eq. (7). Assuming lateral symmetry, only $H_{1}(s)$ and $H_{3}(s)$ need to be specified, corresponding to inputs at FL and RL respectively. Additionally, due to reciprocal relations, in fact only the last two elements of $H_{3}(s)$ need to be specified ${ }^{12}$. In addition to these, the user must also specify the baseline suspension stiffness and damping properties, in case they have to be changed later.

Given this information, the following Matlab code uses new suspension stiffness and damping properties, unsprung mass and tyre compliance properties (stiffness and damping), as well as the desired order of the final reduced order model. The code returns the reduced order model $G_{\text {red }}(s)$, compares the frequency response (Bode plot) and the time domain step response of $G_{r e d}(s)$ with the original $G_{11}(s)$ to help the user decide if a higher order approximation is needed. The user can change suspension properties at will and can simulate response to other excitations beyond a simple step input, if desired.

The transparent algorithmic approach for obtaining this approximation to the quarter-car response, accounting for vehicle flexibility and unsprung mass as well as other wheel effects, is the main contribution of this paper.

\footnotetext{
${ }^{12}$ In terms of the code below, $\mathrm{H} 3(1)$ is the same as $\mathrm{H} 1(3)$ by symmetry of $\mathrm{H}$ (reciprocal theorem), and $\mathrm{H} 3(2)$ is the same as $\mathrm{H} 2$ (3) for the same reason. But H2 (3) is the RL response to FR forcing, which by lateral symmetry of the car is identical to RR response to FL forcing, namely $\mathrm{H} 1$ (4).
} 


\section{Matlab code}

$\%$ Declare ' $s$ ' to be symbolic (Note: with s symbolic, we can define quantities like $0.2(\mathrm{~s}-1) /(\mathrm{s}+3)$, $\%$ save them, load them, etc.) syms $\mathrm{s}$

$\%$ Load $4 \times 1$ transfer function matrices $\mathrm{H} 1(\mathrm{~s})$ and $\mathrm{H} 3(\mathrm{~s})$ respectively

load H1.mat; load H3.mat;

$\%$ Assemble $\mathrm{H}(\mathrm{s})$ :

$\mathrm{H}=[\mathrm{H} 1(1) \mathrm{H} 1(2) \mathrm{H} 3(1) \mathrm{H} 1(4)$;

H1 (2) H1(1) H3(2) H1(3);

$\mathrm{H} 1(3) \mathrm{H} 1(4) \mathrm{H} 3(3) \mathrm{H} 3(4)$;

H1 (4) H1(3) H3(4) H3(3)];

\% Enter baseline (old) suspension properties (see first row of Table 2)

$\mathrm{K}_{-} f=25 ; \mathrm{K}_{-} \mathrm{r}=30 ; \mathrm{C}_{-} f=0.5 ; \mathrm{C}_{-} \mathrm{r}=0.75$;

$\%$ Diagonal old suspension property matrix Do(s)

Do $=\operatorname{diag}\left(\left[K_{-} f+C_{-} f * S ; K_{-} f+C_{-} f * S ; K_{-} r+C_{-} r * S ; K_{-} r+C_{-} r * s\right]\right)$

$\%$ Modifications begin (change below as needed)

$\%$ Input new suspension properties (see second row of Table 2)

$\mathrm{K}_{-} \mathrm{fn}=23.6 ; \mathrm{K}_{-} \mathrm{rn}=34.9 ; \mathrm{C}_{-} \mathrm{fn}=.62 ; \mathrm{C}_{-} \mathrm{rn}=.76$;

$\%$ Diagonal new suspension property matrix Dn(s)

$\mathrm{Dn}=\operatorname{diag}\left(\left[\mathrm{K}_{-} f \mathrm{fn}+\mathrm{C}_{-} \mathrm{fn} * \mathrm{~s} ; \mathrm{K}_{-} \mathrm{fn}+\mathrm{C}_{-} \mathrm{fn} * \mathrm{~s} ; \mathrm{K}_{-} \mathrm{rn}+\mathrm{C}_{-} \mathrm{rn} * \mathrm{~s} ; \mathrm{K}_{-} \mathrm{rn}+\mathrm{C}_{-} \mathrm{rn} * \mathrm{~s}\right]\right)$;

$\%$ Obtaining the modified transfer function matrix Hn(s) from Equation (18)

$\mathrm{Hn}=\left(\left(\mathrm{H}^{\wedge}-1-\mathrm{Do}+\mathrm{Dn}\right)^{\wedge}-1\right) * \mathrm{Dn}$;

$\%$ Input unsprung mass model parameters

M_uf $=45 ; M_{-}$ur $=50$;

$\%$ Input tyre stiffness and damping

$\mathrm{K}_{-} \mathrm{tf}=250 ; \mathrm{C}_{-} \mathrm{tf}=0.05 ; \mathrm{K}_{-} \mathrm{tr}=275 ; \mathrm{C}_{-} \mathrm{tr}=0.06 ;$

$\%$ Assembling the diagonal unsprung mass matrix

M_unsp $=\operatorname{diag}\left(\left[M_{-} u f, M_{-}\right.\right.$uf, M_ur, M_ur $\left.]\right)$;

$\%$ Diagonal tyre compliance matrix Dt(s)

$\mathrm{Dt}=\operatorname{diag}\left(\left[\mathrm{K}_{-} \mathrm{tf}+\mathrm{C}_{-} \mathrm{tf} * \mathrm{~s} ; \mathrm{K}_{-} \mathrm{tf}+\mathrm{C}_{-} \mathrm{tf} * \mathrm{~s} ; \mathrm{K}_{-} \mathrm{tr}+\mathrm{C}_{-} \mathrm{tr} * \mathrm{~s} ; \mathrm{K}_{-} \mathrm{tr}+\mathrm{C}_{-} \mathrm{tr} * \mathrm{~s}\right]\right)$;

$\%$ Obtaining $A(s)$ and $B(s)$ as defined in Equation (23)

$\mathrm{C}=$ M_unsp*s`2+ Dn $+\mathrm{Dt} ; \%$ Intermediate calculation quantity

$\mathrm{A}=\mathrm{C}^{\wedge}-1 * \mathrm{Dn} ; \mathrm{B}=-\mathrm{C}^{\wedge}-1 * \mathrm{Dt}$;

$\%$ Obtaining the final transfer function matrix $G(s)$ from Equation (25)

$\mathrm{G}=\left(\mathrm{A}-\mathrm{Hn} n^{\wedge}-1\right)^{\wedge}-1 * \mathrm{~B}$;

$\%$ Reducing the order of $\mathrm{G}_{-} 11(\mathrm{~s})$ for base excitation only at front left wheel

$[\mathrm{Num}, \mathrm{Den}]=\operatorname{numden}(\mathrm{G}(1,1))$;

$\mathrm{N} 1=\operatorname{double}(\operatorname{coeff} \mathrm{S}(\mathrm{Num})) ; \mathrm{N}=\mathrm{flipud}(\mathrm{N} 1)$

$D 1=\operatorname{double}(\operatorname{coeffS}(\operatorname{Den})) ; D=f l i p u d(D 1) ;$

$\%$ Building a transfer function from numerator and denominator coeffs

$\mathrm{G}_{-} 11=\operatorname{tf}(\mathrm{N}, \mathrm{D})$

$\%$ Defining balred options

opt = balredOptions ('StateElimMethod' , 'MatchDC');

$\%$ Input the desired reduced model order

$\mathrm{n}=6$;

$\%$ Obtaining the reduced order transfer function $\mathrm{G}_{-}$red(s) using balred

$\mathrm{G}_{-}$red $=$balred $\left(\mathrm{G}_{-} 11, \mathrm{n}, \mathrm{opt}\right)$;

$\%$ Extracting the reduced order transfer function coefficients

$\left[N_{-}\right.$red, D_red $]=\operatorname{tfdata}\left(G_{-} r e d\right.$, 'vec');

$\%$ Dropping the sixth order numerator term to obtain a strictly proper TF N_red $=$ N_red $(2:$ end $) ;$ G_red $=$ tf (N_red, D_red);

$\%$ Comparing the bode plots and the step response

figure(1) bode(G_red, ' $r$ ',G_11, '--') ; figure(2) step (G_red, 'r', G_11, '-- ',2) 
This Matlab code can be used to obtain the generalized quarter-car model. It takes only a few seconds to run on an ordinary desktop PC. It enables easy parametric studies where a user can see the effect of changing any parameters of interest on the response of the vehicle. We will now discuss two potential applications.

\section{Applications of our model}

The first is a study on the effect of suspension parameters and second involves wheel hop.

\subsection{Parametric study of suspension}

Here we consider the effects of varying three different parameters.

10.1a Front stiffness: The front suspension stiffness is increased by $25 \%$ and then $50 \%$ from its baseline value $K_{f}^{n}=23.6 \mathrm{~N} / \mathrm{mm}$ (table 2). The corresponding frequency and time domain responses are compared in figure 12 .
As the suspension stiffness increases, the peak overshoot in the unit step response increases because of the reduction in effective damping. More interestingly, note that the steady-state displacement changes slightly as well. In all three cases considered, the steady-state displacement is close to 0.8. For a quarter-car model, the steady-state displacement in response to a unit step displacement input is unity independent of the stiffness. The difference observed here from unity is a strictly otherwheel effect.

10.1b Front damping: The front suspension damping coefficient is increased by $50 \%$ and then $100 \%$ from its baseline value $C_{f}^{n}=0.62 \mathrm{Ns} / \mathrm{mm}$ (table 2). The corresponding frequency and time domain responses are compared in figure 13. With increase in damping, the resonant peak in the Bode plot gets suppressed, and transients in the step response die out faster, as expected.

10.1c Rear damping: Finally, the rear suspension damping coefficient is increased by $50 \%$ and then $100 \%$ from its baseline value $C_{r}^{n}=0.76 \mathrm{Ns} / \mathrm{mm}$ (see table 2 ). The corresponding frequency and time domain responses are
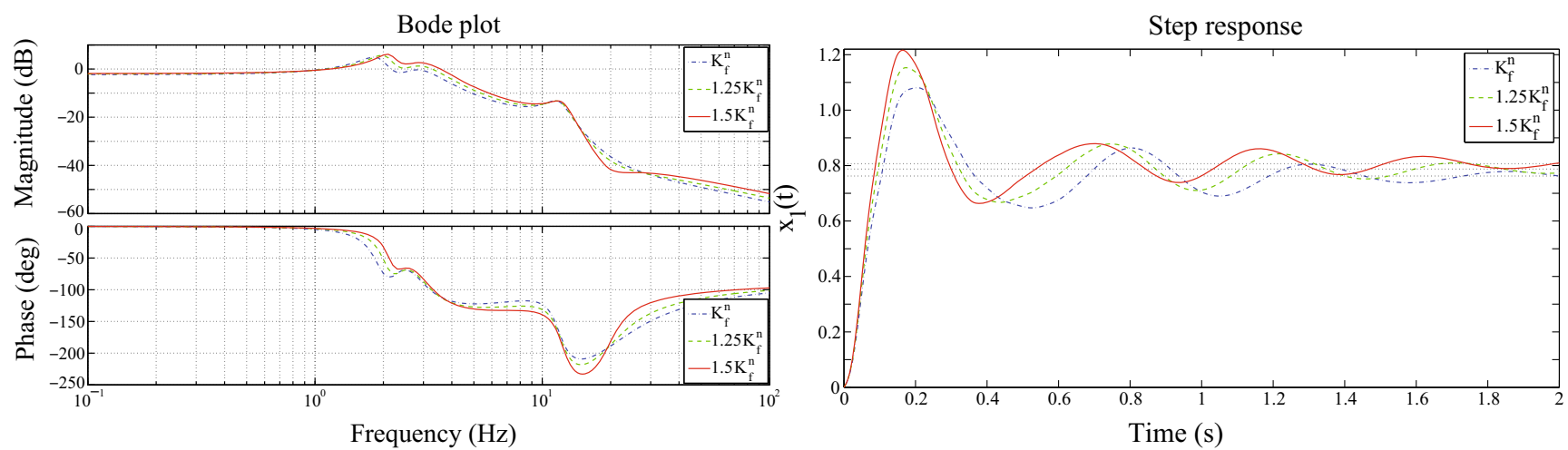

Figure 12. Comparison of frequency domain response (Bode plot: left) and time domain response (step response: right) for different values of front suspension stiffness.
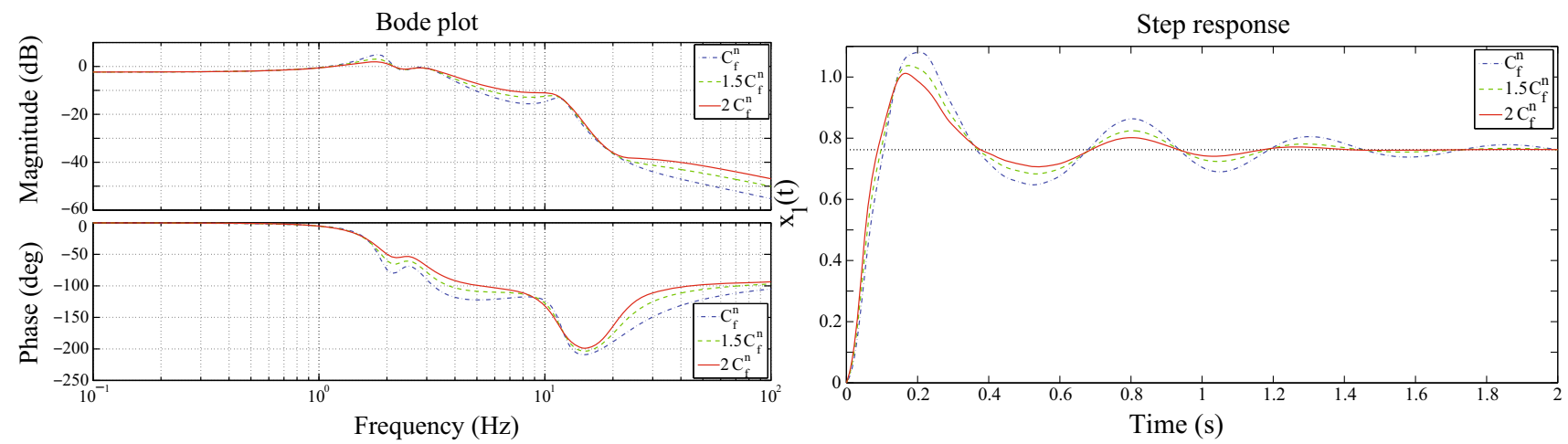

Figure 13. Comparison of frequency domain response (Bode plot: left) and time domain response (step response: right) for different values of front suspension damping coefficients. 

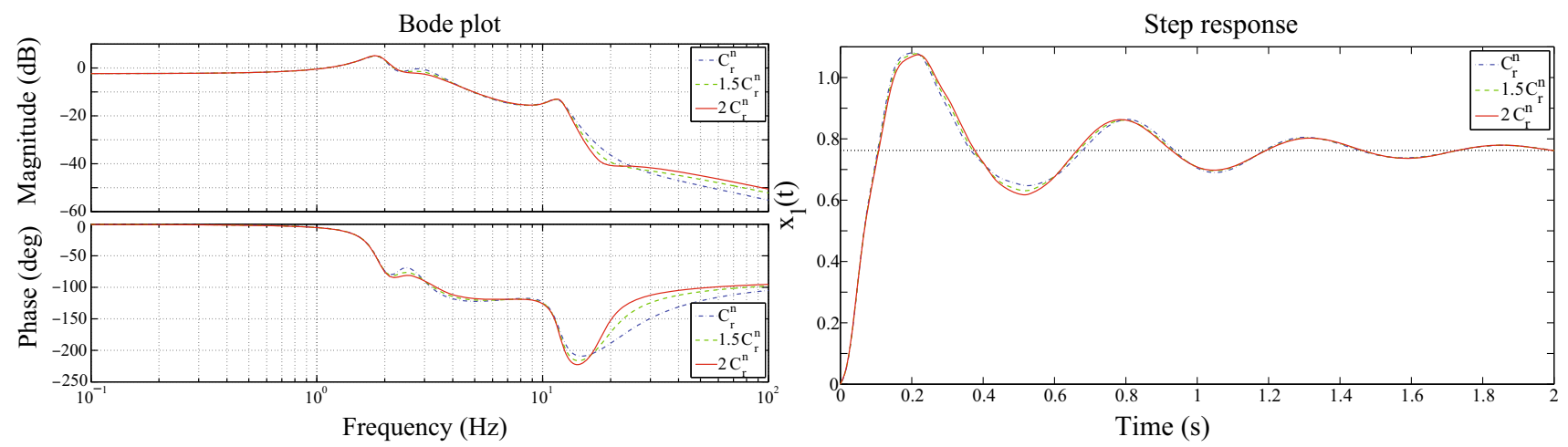

Figure 14. Comparison of frequency domain response (Bode plot: left) and time domain response (step response: right) for different values of rear suspension damping coefficients.

compared in figure 14. As damping increases, the second resonant peak in the Bode plot gets suppressed and the step response shows small changes as well. They are again other-wheel effects, not observed in a usual quarter-car model.

Note also that there is no change in the steady-state displacement due to changes in damping: this shows that our automated approximation methods above retain the 'DC gain' behaviour correctly.

Table 4. Comparison of wheel hop frequencies estimated from Eq. (27) and obtained from our model $G_{\text {red }}(s)$.

\begin{tabular}{lcc}
\hline $\begin{array}{l}\text { Unsprung mass } \\
M_{u f}\end{array}$ & $\begin{array}{c}\text { Quarter-car model } \\
(\text { Eq. }(27))\end{array}$ & $\begin{array}{c}\text { Our model (from poles } \\
\left.\text { of } G_{\text {red }}(s)\right)\end{array}$ \\
\hline $\begin{array}{c}33.75 \mathrm{~kg}(75 \% \text { of } \\
\text { baseline) }\end{array}$ & $14.3 \mathrm{~Hz}$ & $13.2 \mathrm{~Hz}$ \\
$45 \mathrm{~kg}$ (baseline) & $12.4 \mathrm{~Hz}$ & $11.8 \mathrm{~Hz}$ \\
$56.25 \mathrm{~kg}(125 \%$ of & $11.1 \mathrm{~Hz}$ & $10.9 \mathrm{~Hz}$ \\
$\quad$ baseline) & & \\
\hline
\end{tabular}

\subsection{Effect of wheel hop}

Wheel hop is a strong vertical oscillation of the wheels of a car, i.e., a response dominated by motion of the unsprung mass. The particular frequency at which wheel hop may be dominant depends on suspension parameters and unsprung mass but not significantly on vehicle mass. Here we examine the effect of varying the unsprung mass.

The unsprung mass is generally much smaller than the sprung mass; hence, in a simplified quarter-car analysis, the sprung mass is held stationary while estimating the wheel hop frequency [28].

Mimicking that approach in our system, for the FL wheel, the total restoring stiffness is $K_{t f}+K_{f}^{n}$. In this way, an approximate equation of motion for the free vibration of the front wheel assembly is

$$
M_{u f} \ddot{u}+\left(C_{t f}+C_{f}^{\mathrm{n}}\right) \dot{u}+\left(K_{t f}+K_{f}^{\mathrm{n}}\right) u=0,
$$

where $u$ is the corresponding unsprung mass displacement.

The wheel hop frequency is thus estimated to be

$$
\omega_{n} \approx \frac{1}{2 \pi} \sqrt{\frac{K_{t f}+K_{f}^{n}}{M_{u f}}} .
$$
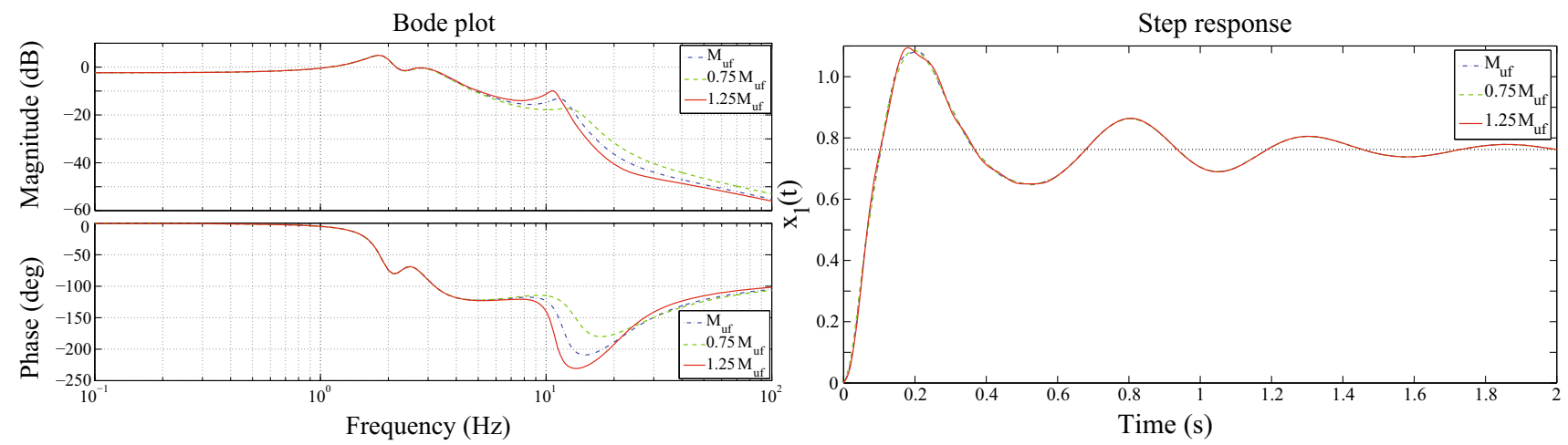

Figure 15. Comparison of frequency domain response (Bode plot: left) and time domain response (step response: right) for different values of the unsprung mass. 
The FOX vehicle that motivates our numerical example has in-hub wheel motors [29], and hence the unsprung mass is a bit higher than for conventional wheels (see table 3). We now study the effect of varying the unsprung mass. Results are given in table 4 and figure 15.

It was observed that in each case, the actual frequency corresponding to poles of $G_{r e d}(s)$ is slightly lower than the frequency estimated using Eq. (27). The actual poles are expected to be slightly lower because of both damping as well as small motions of the sprung mass. Thus, it is seen that wheel hop effects on the body motion are also captured easily by the present approach.

\section{Conclusions}

The vehicle dynamics research literature includes many studies of vehicle suspensions under the quarter-car or half-car simplifications. Here, we have proposed a fairly simple way to incorporate vehicle mass, flexibility and damping effects, as well as the effects of stationary ground contacts at other wheels. In this way, our approach is of intermediate complexity, between those of quarter-car or half-car models on one hand and full-car models on the other. Some obvious differences between usual quarter-car model predictions and our more realistic approach can be seen easily, e.g., in unit step input responses (see Appendix D).

We have also provided a simple Matlab code, which makes it easy for a user to carry out the full range of computations, beginning with Laplace-transformed stepforce input responses from two forcing locations.

In our approach the usual suspension parameters are retained as free parameters to enable semi-detailed comparative studies in the design stage. The model can also be used for the analysis of wheel hop.

In future work, it should be possible to extend or adapt this formulation to incorporate test results in place of initial simulation results, to incorporate more sophisticated unsprung mass models, and also, if there is a need, to incorporate nonlinearities in the localwheel suspension (where displacements are largest), while retaining linear behaviour at other locations. We hope to investigate one or more of these aspects in future research.

\section{Appendix A: Adams suspension model}

The vehicle has independent front and rear suspensions. Suspension modelling is done in Adams Car (figure 16 (top)).

Essential aspects are described here, and some further details are available in [15]. The front suspension is of double wishbone type along with bi-articulated push rods and bell crank levers. The rear suspension is of double wishbone type. Both front and rear suspensions have elastic (spring) and dissipative (hydraulic damper) elements.

An equivalent stiffness and damping need to be estimated for the suspension, accounting for the kinematics of the linkage (i.e., the damper compression differs from that of actual wheel travel). The equivalent suspension stiffness at wheels (the 'wheel rate') was determined directly by a quasistatic wheel travel study of a half-car model in Adams. The equivalent damping coefficient was obtained by scaling the manufacturer-supplied damper data by the motion ratio factor (force and velocity were scaled in inverse proportions) to account for the linkage kinematics (see figure 16 (bottom)). The baseline suspension properties obtained are shown in table 5; see also table 2 in the main text.

\section{Appendix B: Initial estimates of $\sigma \mathrm{S}$ and $\omega$ s for use in section 4}

Here we discuss how to obtain initial estimates of $\sigma \mathrm{s}$ and $\omega \mathrm{s}$ for use in section 4 . We begin with an incremental model with four-dimensional state vector $\bar{z}_{k}$ given by

$$
\bar{z}_{k}=x_{k+1}-x_{k},
$$

where $x_{k+1}$ and $x_{k}$ are the body points' displacements at discrete time intervals $(k+1) T$ and $k T$, respectively. We then extend the dimension of the state vector considered by taking multiple time steps as in

$$
z_{k}=\left[\begin{array}{c}
\bar{z}_{k} \\
\bar{z}_{k+1} \\
\vdots \\
\bar{z}_{k+(j-1)}
\end{array}\right],
$$

where $j$ is to be chosen large enough to get a good fit.

An underlying discrete-time model is assumed in the form

$$
z_{k+1}=D z_{k}
$$

where $D$ is a state transition matrix to be fitted, in a least squares sense, from many $x$-data points. The eigenvalues of $D$ provide the needed estimates of $\sigma \mathrm{s}$ and $\omega \mathrm{s}$, through

$$
\sigma+i \omega=\log _{\mathrm{e}} \frac{\operatorname{eig}(\mathrm{D})}{T},
$$

where $T$ is the time step (in our Adams simulations, $T=0.02 \mathrm{~s}$ ).

The exponential rates obtained for various numbers of retained complex eigenvalues are reported in table 6.

These $\sigma$ s and $\omega$ s were used as initial guesses in nonlinear fitting based on Matlab's built-in optimization routine fminsearch. 

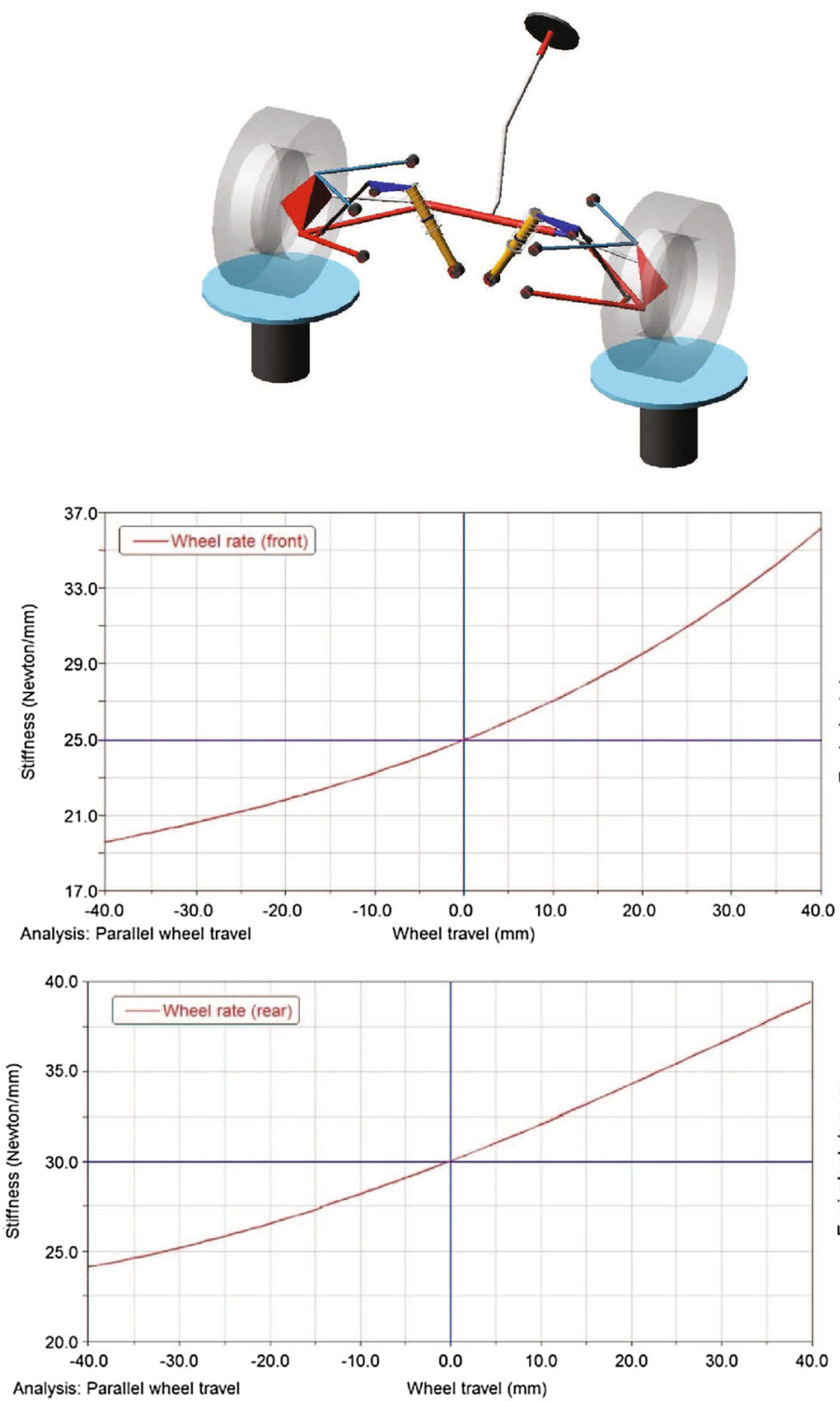
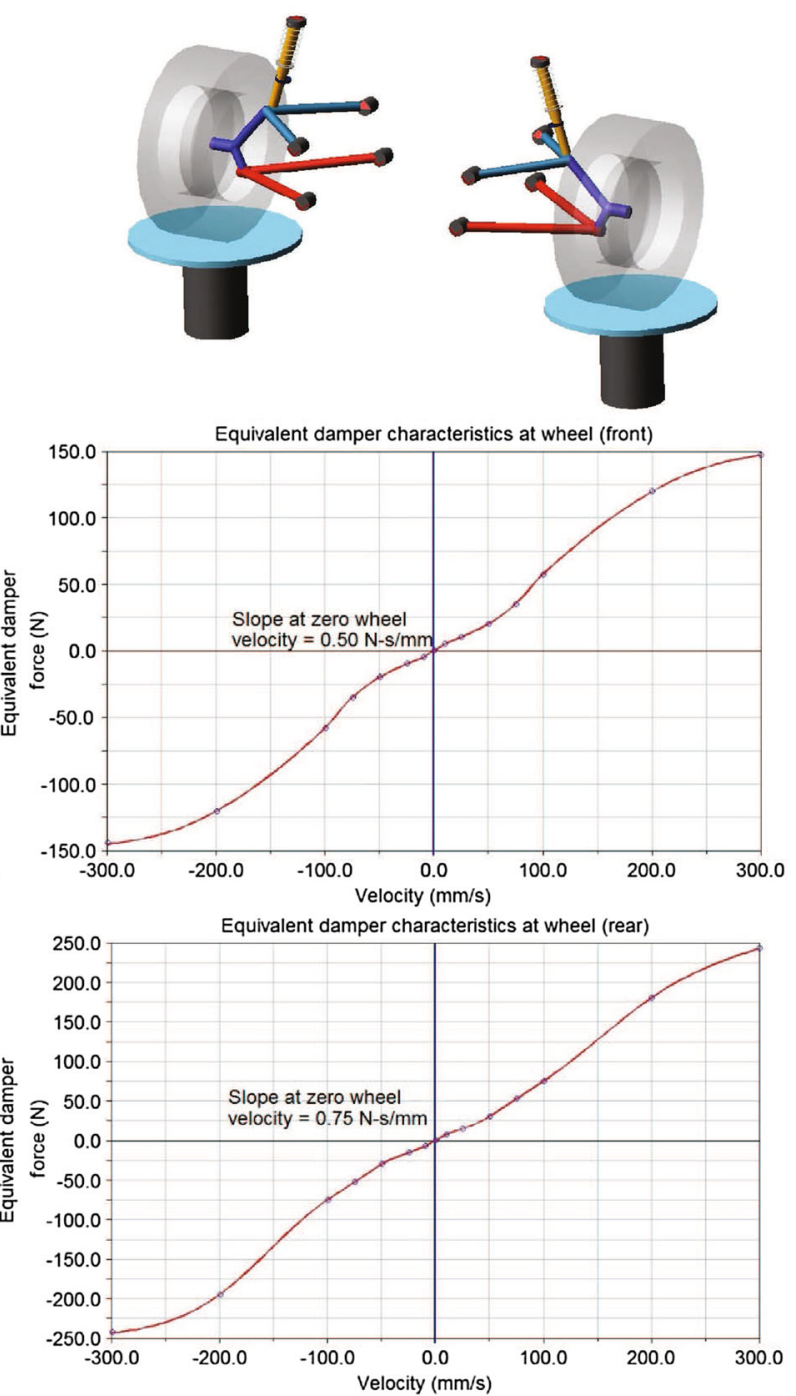

Figure 16. Top: Front and rear suspension assemblies modelled in Adams Car. Bushings connect the suspension to chassis pivot points. Bottom: Equivalent stiffness and damping characteristics of front and rear suspensions, respectively.

Table 5. Initial estimates of suspension parameters.

\begin{tabular}{lccc}
\hline$K_{\text {front }}$ & $K_{\text {rear }}$ & $C_{\text {front }}$ & $C_{\text {rear }}$ \\
\hline $25 \mathrm{~N} / \mathrm{mm}$ & $30 \mathrm{~N} / \mathrm{mm}$ & $0.50 \mathrm{Ns} / \mathrm{mm}$ & $0.75 \mathrm{Ns} / \mathrm{mm}$ \\
\hline
\end{tabular}

\section{Appendix C: Expressions for full and reduced order transfer functions}

$G_{11}(s)$ was found to be the following $36^{\text {th }}$ order transfer function:
Table 6. Initial guesses of $\sigma \mathrm{s}$ and $\omega$. They need not be very accurate, as subsequent nonlinear fitting is done.

No. of complex pairs retained

Identifier

Single-time-step $\quad 2.11 \quad 14.72$ model

Two

Two-time-step model $\quad 1.88 \quad 17.88$

Three

Three-time-step

$1.90 \quad 12.26$

model

$\begin{array}{ll}1.52 & 12.28\end{array}$

$2.41 \quad 18.29$

$4.29 \quad 13.40$ 


$$
\begin{aligned}
& 5.9 s^{35}+3.0 \mathrm{e} 5 s^{34}+5.5 \mathrm{e} 9 s^{33}+5.3 \mathrm{e} 13 s^{32}+1.7 \mathrm{e} 17 s^{31}+3.1 \mathrm{e} 19 s^{30}+8.5 \mathrm{e} 21 s^{29}+1.0 \mathrm{e} 24 s^{28}+1.5 \mathrm{e} 26 s^{27} \\
+ & 1.5 \mathrm{e} 28 s^{26}+1.3 \mathrm{e} 30 s^{25}+9.9 \mathrm{e} 31 s^{24}+6.3 \mathrm{e} 33 s^{23}+3.4 \mathrm{e} 35 s^{22}+1.6 \mathrm{e} 37 s^{21}+6.7 \mathrm{e} 38 s^{20}+2.3 \mathrm{e} 40 s^{19}+7.6 \mathrm{e} 41 s^{18} \\
+ & 2.1 \mathrm{e} 43 s^{17}+5.3 \mathrm{e} 44 s^{16}+1.2 \mathrm{e} 46 s^{15}+2.5 \mathrm{e} 47 s^{14}+4.7 \mathrm{e} 48 s^{13}+7.9 \mathrm{e} 49 s^{12}+1.2 \mathrm{e} 51 s^{11}+1.7 \mathrm{e} 52 s^{10}+2.1 \mathrm{e} 53 s^{9} \\
& +2.4 \mathrm{e} 54 s^{8}+2.5 \mathrm{e} 55 s^{7}+2.3 \mathrm{e} 56 s^{6}+1.8 \mathrm{e} 57 s^{5}+1.3 \mathrm{e} 58 s^{4}+7.6 \mathrm{e} 58 s^{3}+3.9 \mathrm{e} 59 s^{2}+1.3 \mathrm{e} 60 s+4.1 \mathrm{e} 60 \\
G_{11}(s)= & s^{36}+6.2 \mathrm{e} 04 s^{35}+1.4 \mathrm{e} 09 s^{34}+1.6 \mathrm{e} 13 s^{33}+9.2 \mathrm{e} 16 s^{32}+1.3 \mathrm{e} 19 s^{31}+3.3 \mathrm{e} 21 s^{30}+3.5 \mathrm{e} 23 s^{29}+4.7 \mathrm{e} 25 s^{28}+3.8 \mathrm{e} 27 s^{27} \\
+ & 3.3 \mathrm{e} 29 s^{26}+2.1 \mathrm{e} 31 s^{25}+1.3 \mathrm{e} 33 s^{24}+6.4 \mathrm{e} 34 s^{23}+3.0 \mathrm{e} 36 s^{22}+1.1 \mathrm{e} 38 s^{21}+4.0 \mathrm{e} 39 s^{20}+1.2 \mathrm{e} 41 s^{19}+3.3 \mathrm{e} 42 s^{18} \\
+ & 8.3 \mathrm{e} 43 s^{17}+1.9 \mathrm{e} 45 s^{16}+3.9 \mathrm{e} 46 s^{15}+7.3 \mathrm{e} 47 s^{14}+1.2 \mathrm{e} 49 s^{13}+2.0 \mathrm{e} 50 s^{12}+2.8 \mathrm{e} 51 s^{11}+3.7 \mathrm{e} 52 s^{10}+4.3 \mathrm{e} 53 s^{9} \\
+ & 4.7 \mathrm{e} 54 s^{8}+4.5 \mathrm{e} 55 s^{7}+4.0 \mathrm{e} 56 s^{6}+3.0 \mathrm{e} 57 s^{5}+2.0 \mathrm{e} 58 s^{4}+1.1 \mathrm{e} 59 s^{3}+5.6 \mathrm{e} 59 s^{2}+1.8 \mathrm{e} 60 s+5.4 \mathrm{e} 60
\end{aligned}
$$

This higher order transfer function was reduced, using

Matlab's function balred, to

$$
G_{r e d}(s)=\frac{8.3 \mathrm{e}-3 s^{6}+1.1 s^{5}+2.1 \mathrm{e} 2 s^{4}+2.6 \mathrm{e} 4 s^{3}+1.1 \mathrm{e} 6 s^{2}+8.9 \mathrm{e} 6 s+1.9 \mathrm{e} 8}{s^{6}+2.9 \mathrm{e} 1 s^{5}+6.2 \mathrm{e} 3 s^{4}+7.6 \mathrm{e} 4 s^{3}+2.8 \mathrm{e} 6 s^{2}+1.3 \mathrm{e} 7 s+2.5 \mathrm{e} 8} .
$$

The ' $s$ ' term in the numerator of the above transfer function was dropped to obtain a strictly proper transfer function as

$$
G_{r e d}(s)=\frac{1.1 s^{5}+2.1 \mathrm{e} 2 s^{4}+2.6 \mathrm{e} 4 s^{3}+1.1 \mathrm{e} 6 s^{2}+8.9 \mathrm{e} 6 s+1.9 \mathrm{e} 8}{s^{6}+2.9 \mathrm{e} 1 s^{5}+6.2 \mathrm{e} 3 s^{4}+7.6 \mathrm{e} 4 s^{3}+2.8 \mathrm{e} 6 s^{2}+1.3 \mathrm{e} 7 s+2.5 \mathrm{e} 8} .
$$

\section{Appendix D: Further comparison between quarter car and our model}

In this section we compare the unit step response of our model with that of a quarter-car model. Note here that the value of steady-state displacement of a quarter-car model for a unit step displacement input will necessarily be unity. In our model the difference observed from unity (figure 17) is strictly due to other-wheel effects. We have used the FL suspension to body point $B_{1}$ 's displacement $x_{1}(t)$ for comparison. The parameters for building the quarter-car model are reported in table 7 . The vehicle total sprung mass
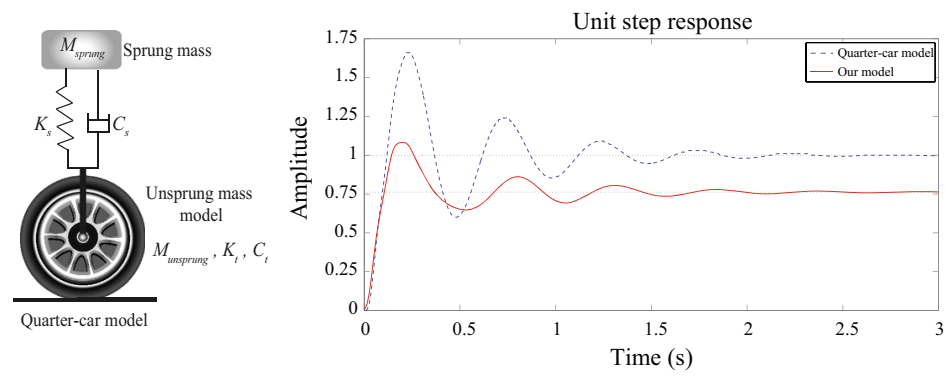

Figure 17. Comparison of displacements obtained from usual quarter-car model and our model in response to a unit step displacement input at wheel contact point $C_{1}$. Both the damping level and steady-state response are affected by nonlocal stiffness and dissipation, which is captured well by our approach. 
Table 7. Quarter-car model parameters.

\begin{tabular}{lccccc}
\hline $\begin{array}{l}M_{\text {sprung }} \\
(\mathrm{kg})\end{array}$ & $\begin{array}{c}M_{\text {unsprung }} \\
(\mathrm{kg})\end{array}$ & $\begin{array}{c}K_{s} \\
\mathrm{~N} / \mathrm{mm}\end{array}$ & $\begin{array}{c}K_{t} \\
\mathrm{~N} / \mathrm{mm}\end{array}$ & $\begin{array}{c}C_{s} \\
\mathrm{Ns} / \mathrm{mm}\end{array}$ & $\begin{array}{c}C_{t} \\
\mathrm{Ns} / \mathrm{mm}\end{array}$ \\
\hline 137 & 45 & 23.6 & 250 & 0.62 & 0.05 \\
\hline
\end{tabular}

is $548 \mathrm{~kg}$. There is almost equal mass distribution between front and rear wheels and the vehicle is assumed to be symmetric about its centre line. Therefore, the sprung mass in the quarter-car model is taken to be $548 / 4=137 \mathrm{~kg}$.

\section{References}

[1] Hrovat D 1997 Survey of advanced suspension developments and related optimal control applications. Automatica 33(10): 1781-1817

[2] Türkay S and Akçay H 2005 A study of random vibration characteristics of the quarter-car model. J. Sound Vib. 282(1): 111-124

[3] Gopala Rao L V V and Narayanan S 2008 Preview control of random response of a half-car vehicle model traversing rough road. J. Sound Vib. 310(1): 352-365

[4] Bouazara M and Richard M J 2001 An optimization method designed to improve 3-D vehicle comfort and road holding capability through the use of active and semi-active suspensions. Eur. J. Mech.-A/Solids 20(3): 509-520

[5] Kim C and Ro P I 2002 An accurate full car ride model using model reducing techniques. J. Mech. Des. 124(4): 697-705

[6] Ersal T, Kittirungsi B, Fathy H K and Stein J L 2009 Model reduction in vehicle dynamics using importance analysis. Veh. Syst. Dyn. 47(7): 851-865

[7] Li K, Zheng S, Yang D, Lian X and Nagai M 2003 Modeling and control of active suspensions for MDOF vehicle. Tsinghua Sci. Technol. 8(2): 224-230

[8] Ibrahim I M, Crolla D A and Barton D C 1996 Effect of frame flexibility on the ride vibration of trucks. Comput. Struct. 58(4): 709-713

[9] Liang C Y and Peng H 1999 Optimal adaptive cruise control with guaranteed string stability. Veh. Syst. Dyns. 32(4-5): 313-330

[10] Yih P and Gerdes J C 2005 Modification of vehicle handling characteristics via steer-by-wire. IEEE Trans. Control Syst. Technol. 13(6): 965-976
[11] Kanchwala H, Wideberg J, Alba C B and Marcos D 2015 Control of an independent 4WD electric vehicle by DYC method. Int. J. Veh. Syst. Model. Test. 10(2): 168-184

[12] Alleyne A 1997 Improved vehicle performance using combined suspension and braking forces. Veh. Syst. Dyn. 27(4): 235-265

[13] Adams M D Accessed on: 2015-11-10 http://web.mscsoft ware.com/Products/CAE-Tools/MD-Adams.aspx

[14] Grand Tourer (GT) Accessed on: 2015-11-10 http://en.wiki pedia.org/wiki/Grand_tourer

[15] Wideberg J, Bordons C, Luque P, Mántaras D A, Marcos D and Kanchwala H 2014 Development and experimental validation of a dynamic model for electric vehicle with in hub motors. Procedia-Soc. Behav. Sci. 160: 84-91

[16] Papalukopoulos C and Natsiavas S 2007 Nonlinear biodynamics of passengers coupled with quarter car models. $J$. Sound Vib. 304(1): 50-71

[17] Davison E J 1966 A method for simplifying linear dynamic systems. IEEE Trans. Autom. Control 11(1): 93-101

[18] Aoki M 1968 Control of large-scale dynamic systems by aggregation. IEEE Trans. Autom. Control 13(3): 246-253

[19] Chen C F and Shieh L S 1968 A novel approach to linear model simplification. Int. J. Control 8(6): 561-570

[20] Moore B C 1981 Principal component analysis in linear systems: controllability, observability, and model reduction. IEEE Trans. Autom. Control 26(1): 17-32

[21] Kim C and Ro P I 2000 Reduced-order modelling and parameter estimation for a quarter-car suspension system. Proc. Inst. Mech. Eng., Part D: Jo. Automob. Eng. 214(8): 851-864

[22] Negrut D and Dyer A 2004 Adams/solver primer. Ann Arbor: Adams

[23] Barber J R 2004 The reciprocal theorem. In: Solid mechanics and its applications, vol. 107: Elasticity, pp. 393-404. Netherlands: Springer

[24] Lord Rayleigh 1877 The theory of sound, vol. 1. London: Macmilan

[25] Adhikari S and Woodhouse J 2001 Identification of damping: part 1, viscous damping. J. Sound Vib. 243(1): 43-61

[26] Adhikari S and Woodhouse J 2002 Identification of damping: part 3, symmetry-preserving methods. J. Sound Vib. 251(3): 477-490

[27] Healey A 1924 The tyre as part of the suspension system. Proc. Inst. Automob. Eng. 19(1): 26-128

[28] Dixon J 2008 The shock absorber handbook. New York: Wiley

[29] Kelly Controls LLC. Accessed on: 2016-02-15. http://www. kellycontroller.com/car-hub-motor-72v-7kw-p-711.html 